ORIGINAL ARTICLE

\title{
Alterations in the expression of a neurodevelopmental gene exert long-lasting effects on cognitive-emotional phenotypes and functional brain networks: translational evidence from the stress-resilient Ahil knockout mouse
}

\author{
A Lotan ${ }^{1,5}$, T Lifschytz $^{1,5}$, B Mernick ${ }^{1,4}$, O Lory ${ }^{2}$, E Levi $^{3}$, E Ben-Shimol ${ }^{2}$, G Goelman ${ }^{2}$ and B Lerer ${ }^{1}$
}

\begin{abstract}
Many psychiatric disorders are highly heritable and may represent the clinical outcome of early aberrations in the formation of neural networks. The placement of brain connectivity as an 'intermediate phenotype' renders it an attractive target for exploring its interaction with genomics and behavior. Given the complexity of genetic make up and phenotypic heterogeneity in humans, translational studies are indicated. Recently, we demonstrated that a mouse model with heterozygous knockout of the key neurodevelopmental gene Ahi1 displays a consistent stress-resilient phenotype. Extending these data, the current research describes our multi-faceted effort to link early variations in Ahi1 expression with long-term consequences for functional brain networks and cognitive-emotional phenotypes. By combining behavioral paradigms with graph-based analysis of whole-brain functional networks, and then cross-validating the data with robust neuroinformatic data sets, our research suggests that physiological variation in gene expression during neurodevelopment is eventually translated into a continuum of global network metrics that serve as intermediate phenotypes. Within this framework, we suggest that organization of functional brain networks may result, in part, from an adaptive trade-off between efficiency and resilience, ultimately culminating in a phenotypic diversity that encompasses dimensions such as emotional regulation and cognitive function.
\end{abstract}

Molecular Psychiatry (2017) 22, 884-899; doi:10.1038/mp.2016.29; published online 29 March 2016

\section{INTRODUCTION}

Many psychiatric disorders are highly heritable and are likely to represent the clinical outcome of aberrations in the formation of brain architecture in utero or during early postnatal life. ${ }^{1}$ Given their strong genetic underpinnings, ${ }^{2}$ measures of network topology have been implicated as intermediate phenotypes that indicate the genetic risk for neuropsychiatric disorders. ${ }^{3}$ In recent years, graph-theory-based complex network analysis, which describes important properties of complex systems by quantifying topologies of their respective network representations, has been increasingly used in the study of structural and functional organization of the nervous system. ${ }^{4}$ By treating the brain as one integrated network, this analysis asks whether the architecture of communication patterns within that network is altered under various conditions and disorders. ${ }^{5}$

The placement of brain connectivity as an 'intermediate phenotype' positioned between genetics and behavior renders it an attractive target for studies that link networks across levels, from molecules to neurons and brain systems. ${ }^{6}$ Moreover, the emergence of comprehensive data sets on gene expression and brain networks in healthy and affected individuals offers the possibility of addressing new questions regarding the interaction of brain connectivity with genomics and behavior, including its dysregulation, as a possible trigger of brain disorders. ${ }^{7-9}$ However, the complexity of genetic makeup, in conjunction with pronounced phenotypic heterogeneity, greatly complicates such systematic attempts. ${ }^{10}$ Translational studies using inbred or genetically modified mice could help bridge such major gaps. ${ }^{11-14}$

From this perspective, the Abelson helper integration site-1 (Ahi1) knockout mouse emerges as a promising model for gaining mechanistic insights into how functional (dis)organization of mature brain networks, accompanied by corresponding behavioral phenotypes, had been established by earlier developmental abnormalities. Ahi1 protein, Jouberin, is highly expressed in mammals throughout the developing brain, with lower expression continuing into adulthood. ${ }^{15}$ Although conserved in mammals, Jouberin's accelerated evolution in the human lineage indicates a role in cognitive (dys)function. ${ }^{16}$ Recent converging data emphasize Ahi1's extensive developmental role, from essential cellular signaling organelles, such as the primary cilium, to neuronal networks and complex organ systems. ${ }^{17-22}$ Previous work by our group discovered significant association of AHI1 with schizophrenia, ${ }^{23,24}$ which was later replicated in large European casecontrol samples. ${ }^{25,26}$ In addition, associations of $A H I 1$ with autism and mood disorders have been reported. ${ }^{27,28}$ Notably, modest alterations in AHI1 expression have been implicated in both schizophrenia and autism. ${ }^{29,30}$

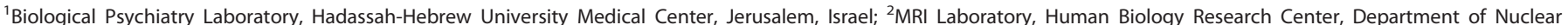

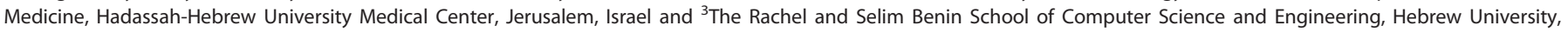
Jerusalem, Israel. Correspondence: Professor B Lerer, Biological Psychiatry Laboratory Hadassah - Hebrew University Medical Center, Jerusalem 91120 , Israel.

E-mail: lerer@hadassah.org.il

${ }^{4}$ Present affiliation: Developmental Psychopathology Laboratory, Department of Psychology, University of Haifa, Haifa, Israel.

${ }^{5}$ These authors contributed equally to this work.

Received 30 July 2015; revised 29 December 2015; accepted 3 February 2016; published online 29 March 2016 
Recently, we performed comprehensive behavioral phenotyping of the heterozygous Ahi1 knockout $\left(A h i 1^{+/-}\right.$) mouse, which displayed diminished, rather than absent, Ahi1 expression. Although we had not observed significant between-genotype differences in physical characteristics or gross brain morphology, we presented consistent evidence implicating underexpression of the Ahi1 gene during neurodevelopment in relative resilience to various stressors during adulthood. ${ }^{31}$ This resilience manifested as an anxiolytic-like phenotype across several paradigms that involved exposure to environmental stress and was accompanied by a blunted response of the autonomic nervous system and the hypothalamic-pituitary-adrenal axis. Based on seed-voxel correlations derived from functional magnetic resonance imaging (fMRI), functional disconnectivity between the amygdala and other brain regions involved in processing of anxiogenic stimuli was suggested as an underlying mechanism for this phenotype. ${ }^{32}$ Although these data contributed to understanding the genetic basis of emotional regulation and its associated neurocircuitry, we did not correlate gene expression and behavior with the global organization of brain networks. Moreover, we focused mainly on emotional phenotypes and treated Ahi1 expression dichotomously rather than as a continuum.

In this paper, we describe a multi-modal effort to link early variations in expression of the neurodevelopmental gene, Ahi1, to long-term effects on cognitive-emotional phenotypes and functional brain networks. In the present report: (i) We add the cognitive-emotional interface to the stress-resilient behavioral phenotypes previously reported in relation to our $\mathrm{Ahil}^{+/-}$mouse, showing that although its cognitive performance is diminished overall, it may have an advantage in highly stressogenic scenarios. Then, by analyzing data from a comprehensive neuroinformatic database of inbred mouse strains, ${ }^{33}$ we corroborate and extend the behavioral data by (ii) demonstrating that lower neocortical Ahi1 expression during neurodevelopment is associated with diminished capacity for associative learning in adulthood and by (iii) suggesting that the latter may arise from polymorphisms in a trans-acting major regulatory element that modulates the expression of several functionally related genes during neurodevelopment. (iv) Finally, we assess the global topology of whole-brain networks using resting-state fMRI, to reveal that networks derived from $\mathrm{Ahi1}^{+/-}$mice display higher assortativity but lower hierarchy compared with the $A h i 1^{++}$networks.

\section{MATERIALS AND METHODS}

Generation of the $\mathrm{Ahi}^{+/-}$mouse line

As previously described, chimeric male mice carrying a gene trap vector ${ }^{34}$ at the Ahi1 locus were backcrossed with WT C57BL/6 females to generate heterozygous Ahi1 knockout mice $\left(\mathrm{Ahi1}^{+/-}\right) .^{31} \mathrm{Ahi1^{+/- }}$ males were then backcrossed to wild-type $\left(\mathrm{Ahil}^{+/+}\right)$females for over eight generations in order to achieve homogenous background. As previously reported, Ahi ${ }^{+/-}$mice displayed a $\sim 50 \%$ reduction in Jouberin (Ahi1 protein) levels at postnatal day $3{ }^{31}$ corresponding to the neurodevelopmental window when Jouberin is most critical. ${ }^{35}$ This between-genotype difference was no longer present in adulthood, when Ahi1 expression falls dramatically. For the current experiments, we used a wild-type $x$ heterozygous breeding scheme. By using littermates as controls, biases due to maternal effects are minimized. All animal procedures were approved by the Hebrew University Institutional Animal Care and Use Committee.

\section{Design of animal experiments}

Prior to behavioral testing, mice were housed at the Hebrew University Reproduction Unit under specific pathogen-free conditions with a 12-h light/dark cycle. Food and water were provided ad libitum. All experiments were conducted during morning hours (0900-1200 hours). Two-month-old male mice underwent the following tests: (i) Open field (OF) and swim-tovisible platform task $(n=25)$; (ii) novel object recognition $(n=16)$; and (iii) fear conditioning $(n=30)$. Experiments were conducted in a fixed order and on separate days using counterbalanced groups. In the behavioral experiments, animals were tracked with a video camera positioned overhead and data were extracted with Ethovision (Noldus Information Technologies, Wageningen, The Netherlands). For the graph-based study of functional brain networks, imaging data that had been previously acquired $^{31}$ was re-analyzed using the relevant mathematical tools $(n=25)$.

\section{Behavioral measures}

Open field. The apparatus consisted of a square arena measuring $50 \times 50 \times 33 \mathrm{~cm}^{3}$ under 15 lux illumination. Mice from both genotypes were allowed to explore the arena for $6 \mathrm{~min}$. Time spent in the central zone of the arena $\left(10 \times 10 \mathrm{~cm}^{2}\right)$ was calculated.

Swim-to-visible platform task. Modified from Beiko et al., ${ }^{36}$ a platform $\left(15 \times 15 \mathrm{~cm}^{2}\right)$ was placed inside the center of a round $(120 \mathrm{~cm}$ diameter) pool, protruding $2 \mathrm{~cm}$ above the surface of the water and marked by a 10$\mathrm{cm}$ tall flag. Each mouse was placed in the pool once and allowed a maximum of $120 \mathrm{~s}$ to locate the platform. Escape latency, distance travelled and percentage of time spent in the peripheral $20 \mathrm{~cm}$ ring were calculated.

Novel object recognition test. In the one-trial non-matching-to-sample object recognition task, ${ }^{37}$ mice were allowed to explore two 'identical' to-be-familiarized (sample) objects for $10 \mathrm{~min}$. After $1 \mathrm{~h}$, mice were returned to the apparatus, which now contained one familiar object and a novel object. Discrimination ratio was defined as the novel object interaction duration divided by the total duration of interaction with both objects during a 5-min test session. As stress and novelty can adversely affect object interaction and hence object recognition, mice were handled daily for 3 days and habituated to the test arena before the experiment.

Fear conditioning. Training was conducted in a conditioning chamber $\left(19 \times 25 \times 19 \mathrm{~cm}^{3}\right)$ equipped with black methacrylate walls, transparent front door, a speaker and grid floor, placed inside an outer soundattenuating chamber (StartFear System, Panlab, Holliston, MA, USA). A computer program (PACKWIN 2.0, Panlab) controlled the audio generator to deliver the tone and the shock generator, wired to the grid floor, which generated a scrambled foot-shock. Following a similar design to that described by Licht et al., ${ }^{38}$ fear conditioning to both context and cue was evaluated $24 \mathrm{~h}$ after the conditioning procedure (see Supplementary Methods for further details).

Measuring the relationship between Ahi1 expression and fear learning across the BXD recombinant inbred strains

We employed GeneNetwork (http://www.genenetwork.org), a website that integrates data sets of complex traits with software for quantifying correlations among traits. ${ }^{3,39}$ The data sets include both biological traitsneuroanatomical, pharmacological, and behavioral—and microarray-based gene expression data from numerous brain regions across recombinant inbred (RI) lines, which were generated by crosses of C57BL/6J and DBA/2 (BXD) inbred strains. Each of the BXD strains is a unique 'mosaic' of chromosomal segments inherited from either the $B$ or $D$ progenitor strain. ${ }^{40} \mathrm{~A}$ search function finds correlations between mRNA expression and biological traits. ${ }^{33}$ Further details depicting the methodology used for curating neocortical Ahi1 expression profiles and fear conditioning traits and then inter-correlating them are provided in the Supplementary Methods and in Supplementary Figure S1.

Mapping QTLs that modulate Ahi1 expression and fear learning across the BXD RI strains

A quantitative trait loci (QTL) is a chromosome region that contains one or more sequence variants that modulate the distribution of a variable trait measured in a sample of genetically diverse individuals from an interbreeding population. Differences in the expression of mRNA are often treated as standard phenotypes, much like behavioral phenotypes. However, QTLs of mRNAs (termed expression QTLs (eQTLs)) have the unique property of (usually) having a single parent gene and genetic location. An eQTL that maps to the location of the parent gene that produces the mRNA is referred to as a cis-eQTL, while an eQTL that maps far away from its parent gene is referred to as trans-eQTL. ${ }^{41}$ One of the most powerful features of GeneNetwork is its ability to detect and evaluate, both qualitatively and quantitatively, common and unique (e) 
QTLs for a set of traits that include both expression and behavior phenotypes. $^{33,42}$ In the current analysis, the following issues were sequentially addressed: (i) eQTL mapping of neocortical Ahi1 expression during the neonatal period; (ii) determining whether the major eQTL regulating neonatal Ahi1 expression also serves as a QTL for associative fear learning in adulthood; (iii) identifying genes within the developing neocortex that share their major eQTL with Ahi1; (iv) enrichment analysis based on these genes; and finally (v) Generation of QTL heat maps loaded with traits that represent both the gene transcripts that comprise the highly enriched Gene Ontology categories identified and the relevant fear conditioning traits. Further details of the methodology used are provided in Supplementary Methods.

\section{Resting-state functional networks}

Acquisition. As previously described, ${ }^{31}$ and in line with a recent report using a similar methodology in rats, ${ }^{43}$ MRI measurements were performed with a 4.7-T Bruker BioSpec scanner (Bruker Biospin, Ettlington, Germany) using a Dotty quadrature mouse head coil (see Supplementary Methods for further details).

Preprocessing. As previously described, ${ }^{31,43}$ functional data were first processed in the SPM8 software (http://www.fil.ion.ucl.ac.uk/spm/software/ spm8) using standard spatial preprocessing steps. Images were slice-time corrected and realigned. Data with motion that was $>0.2 \mathrm{~mm}$ (one voxel) or that needed a rotation $> \pm 0.002^{\circ}$ for their registrations were removed from the analysis. At a second step, analysis was performed using the custom-made IDL and MATLAB software. It included regression-out of the six functions that are related to motion (from the SPM analysis), 2D nonlinear alignment of the MRI images to the mouse brain atlas, ${ }^{44}$ data smoothing by a three-point-Gaussian kernel and band-pass filtering $(<0.01$ to $>0.1 \mathrm{~Hz})$.

Generation of functional brain networks. Based on our previous experience $^{43}$ and current hypothesis, regions of interest (ROIs) scattered throughout the brain were preselected. Representative ROls within the cortices, basal ganglia, limbic system and midbrain were included. Interregional correlations in blood oxygenation-level dependent (BOLD) signal between all ROls were averaged across time using the above-mentioned software. For improving normality, all correlation values underwent a Fisher's $r$-to-z transformation.

Graph-based analysis. For graph-theoretical analysis of neural networks through $\mathrm{fMRl}$, anatomical brain regions are considered as nodes, linked by edges, which represent the connectivity measured by the temporal correlation of BOLD signal fluctuations between the nodes. ${ }^{5}$ The topological properties of the networks derived by thresholding the matrix of inter-regional BOLD signal correlations depend on the choice of threshold values. ${ }^{4}$ Our first effort was therefore to define a range of thresholds that yielded fully connected networks with a small-world topology. ${ }^{45}$ Next, we determined the assortativity ${ }^{46}$ and hierarchy ${ }^{47}$ coefficients for each individual network and compared means between groups $^{48}$ (see Supplementary Methods for further details).

\section{Statistical analysis}

In the behavioral tests, mean scores among $\mathrm{Ahi1}^{+/+}$and $\mathrm{Ahi1}^{+/-}$mice were compared using the non-paired samples $t$-test. In the fear conditioning experiments that involved both genotype and conditioning-state as the independent between- and within- group variables, respectively, mixed analysis of variances were conducted. These were then followed by simple effects analysis, depending on the statistical significance of the interaction and the main effects. ${ }^{49}$ Both Pearson's product-moment and Spearman rank-order correlations were used for estimating relationships between gene expression profiles and behavioral traits obtained from the BXD RI strain database. In the graph-based network analysis, quantification of local and global network metrics was performed using the MATLAB-based graph-theoretical analysis toolbox for analyzing between-group differences in large-scale structural and functional brain networks. ${ }^{48}$ Betweengenotype group comparisons were performed using a bootstrap test for non-paired samples (1000 permutations). Further details of the statistical approaches used are provided in Supplementary Methods.

\section{RESULTS}

Behavioral experiments measuring the cognitive-emotional interface

Besides replicating the previously reported anxiolytic-like phenotype, ${ }^{31}$ these experiments were conducted in order to explore cognitive aspects of the Ahi1 knockout mouse, with an emphasis on the cognitive-emotional interface.

Anxiety and performance during exposure to a novel stressor. When introduced into novel OF and Morris water maze (MWM) arenas, naive $\mathrm{Ahi1}^{+/-}$mice displayed significantly lower thigmotactic behavior compared with naive $A h i 1^{+/+}$mice (OF; $t_{23}=2.7$, $P=0.013$ and MWM; $t_{23}=3.1, P=0.005$, respectively, Figures $1 \mathrm{a}-\mathrm{d}$ ). During first-time MWM exposure, $\mathrm{Ahi}^{+/-}$mice displayed superior performance compared with $\mathrm{Ahi1}^{+/+}$mice in the swim-to-visible platform task, manifested as a shorter latency and a trend for shorter distance to reach platform $\left(t_{23}=2.2, P=0.042\right.$ and $t_{23}=1.9$, $P=0.075$, respectively, Figures $1 \mathrm{e}$ and $\mathrm{f}$ ). Notably, travelling and swimming velocities were similar across genotypes (Figures $1 \mathrm{~g}$ and $\mathrm{h}$ ), indicating that differences in motor abilities did not account for the superior performance of the $A h i 1^{+/-}$mice in this task.

Learning in a low-stress environment. By choosing a small apparatus with which the animals have been familiarized during pretraining, the OF situation which evokes anxiety- and stressrelated behaviors such as thigmotaxis can be minimized. ${ }^{50}$ Under such conditions, the preference of $\mathrm{Ahi1}^{+/-}$mice for the novel object during the novel object recognition test (NORT) was significantly reduced compared with their $\mathrm{Ahil}^{+/+}$littermates, as expressed by the corresponding difference score ( -1.89 vs 4.00 , $\left.t_{8.8}=2.9, P=0.017\right)$ and discrimination ratio (0.44 vs $0.62, t_{8.2}=3.0$, $P=0.016$, Figure 2a).

Associative fear learning. Although passive defensive responses such as freezing are reflexive, fear conditioning paradigms measure the freezing response that takes place following pairing of an unconditioned stimulus, such as foot shock, with a conditioned stimulus. ${ }^{51}$ As such, fear conditioning to either context or cue represents a form of associative learning that has been extensively studied in many species. ${ }^{52}$ Using a mixed analysis of variance design, with conditioning-state (habituation vs training vs context test) and genotype as the within- and between-subject variables, respectively, and freezing to context (percentage) as the dependent variable, a significant interaction was noted $\left(F_{2,56}=8.4, P=0.0006\right.$, Figure $\left.2 \mathrm{~b}\right)$. Post-hoc analysis revealed significant increases in freezing behavior during the immediate postshock period (Bonferroni's pairwise comparisons, mean differences relative to habituation are 20.47 and 19.97 for $\mathrm{Ahil}^{+/+}$and $\mathrm{Ahi1}^{+/-}$mice, respectively, $P<0.0005$ for both genotypes) and then again during the context test (mean differences relative to postshock period are 53.59 and 33.43 for wild-type and $\mathrm{Ahi1}^{+/-}$mice, respectively, $P<0.0005$ for both genotypes). However, analysis of the simple main effect of genotype at each conditioning-state level revealed that, while freezing behavior was similar during habituation and postshock periods, during the context test $A \mathrm{Ai}^{+/-}$mice displayed significantly lower freezing compared with wild-type littermates (independent samples test, $t_{28}=3.1, P=0.005$ ). These results suggest that underlying differences in the innate, footshockelicited, defensive freezing reaction were unlikely to account for the strong between-genotype difference upon context re-exposure. Using a similar design, with exposure to cue (pretone vs tone) and genotype as the within- and between-subject variables, respectively, a significant interaction was also noted $\left(F_{1,28}=8.274, P=0.008\right.$, Figure $\left.2 \mathrm{c}\right)$. Similarly, post-hoc analysis revealed that, while exposure to the cue elicited large increases in freezing in both $A h i 1^{+/+}$and Ahi1 ${ }^{+/-}$mice, a between-genotype 

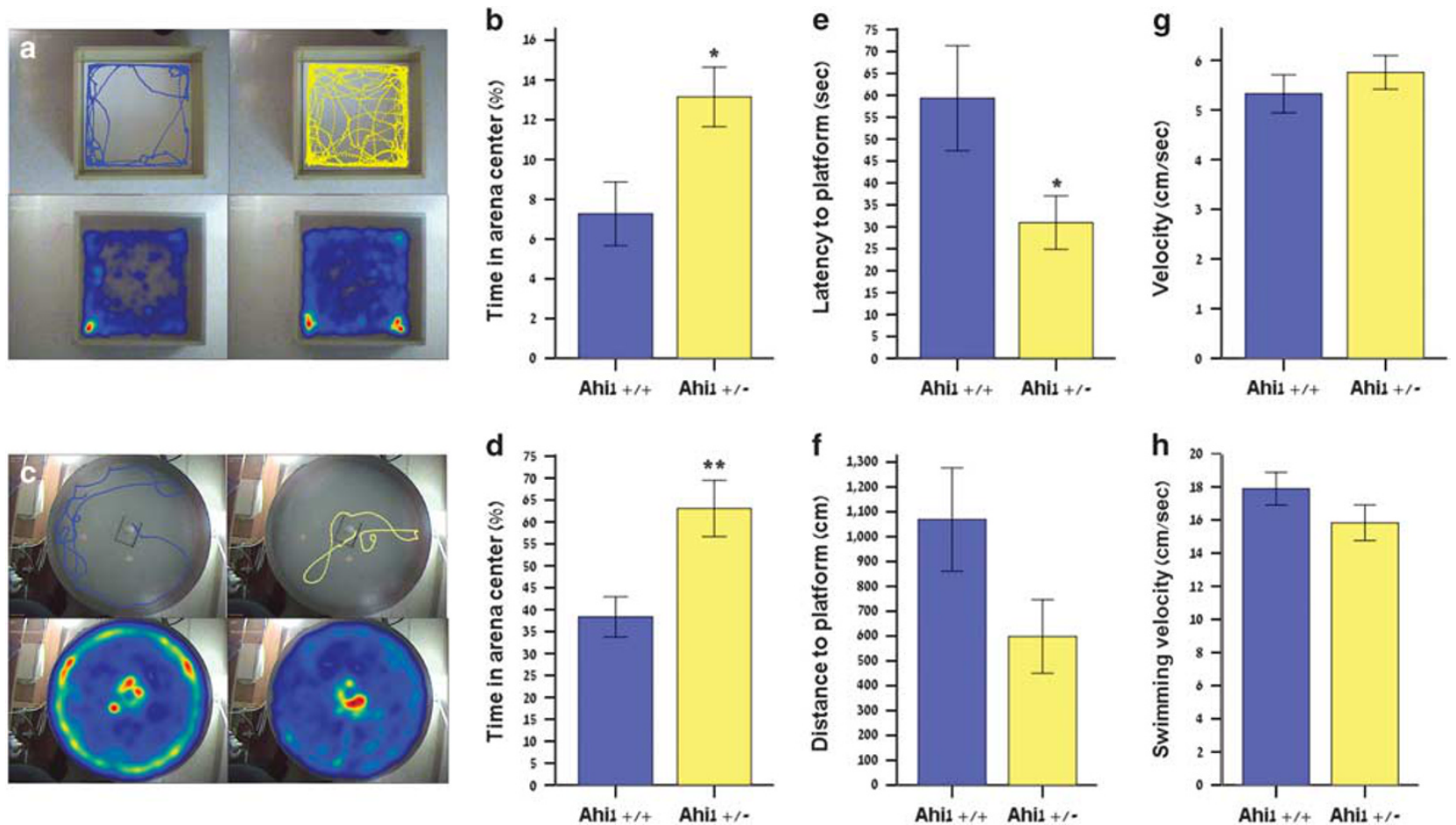

Figure 1. Anxiety vs performance during exposure of the Ahi1 knockout mouse to a novel stressor. (a, $\mathbf{b})$ Thigmotaxis in the open field arena. (a, upper panel) Track visualizations of typical Ahi1 ${ }^{+/+}$(left, blue tracing) and $A$ hi $1^{+/-}$(right, yellow tracing) mice inside the open field arenas. (a, lower panel) Heat map visualizations of $A h i 1^{+/+}$(left) and $A h i 1^{+/-}$(right) mice inside the open field arena. For each genotype, all relevant tracks were merged into one heat map. The color of each pixel represents the mean time subjects located at that specific location. (b) Bar graphs depicting the proportion (percent) of time that mice from each genotype spent in the center of the open field arena. Compared with $A$ hi $1^{+/+}$ mice, $\mathrm{Ahi1}^{+/-}$mice displayed significantly more time in the center when placed in the novel, highly stressogenic open field arena. (c, d) Thigmotaxis in the first-time swim-to-visible platform task inside a Morris water maze (MWM) arena. (c) Track visualizations of typical Ahi1 $1^{+/+}$ and $\mathrm{Ahi}^{+/-}$mice (upper panel) and heat map visualizations of group means (lower panel) in this task are presented as detailed above. (d) Bar graphs depicting the proportion (percentage) of time that mice from each genotype spent in the center of the MWM arena. (e, $\mathbf{f})$ First-time performance in the swim-to-visible platform task. Ahi $1^{+/-}$mice outperformed their Ahi $1^{+/+}$controls in the MWM visible platform escape task, manifested by the latter's longer escape latency (e) and marginally longer distance swim (f). (g, $\mathbf{h})$ Velocities during exposure to the novel stressors. Travelling $(\mathbf{g})$ and swimming $(\mathbf{h})$ velocities were similar across genotypes. ${ }^{*} P<0.05 .{ }^{* *} P<0.01 . n=12\left(\right.$ Ahi $\left.{ }^{+/+}\right), n=13\left(\right.$ Ahi1 $\left.{ }^{+/-}\right)$. Data are expressed as mean \pm s.e.m.
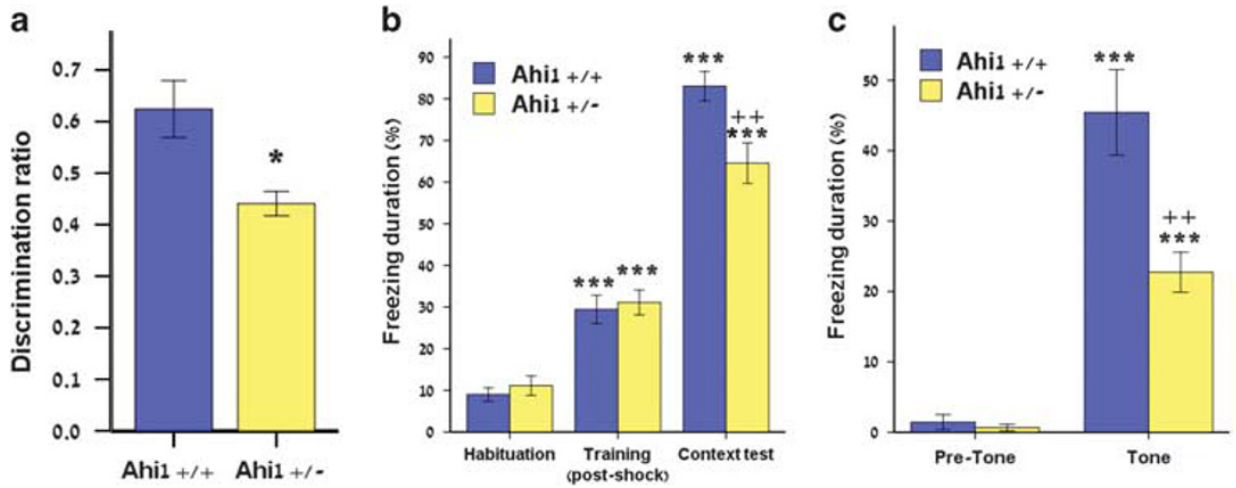

Figure 2. Memory and fear learning in the Ahi knockout mouse. (a) Novel object recognition. In this low-stress task, Ahi1 ${ }^{+/+}$mice displayed better performance than their Ahi $1^{+/-}$littermates, as manifested by their superior discrimination ability (ratio of time spent with the novel object out of total time spent with both objects). ${ }^{*} P<0.05 . n=7\left(A h i 1^{+/+}\right), n=9\left(A h i 1^{+/-}\right)$. (b, c) Pavlovian fear conditioning. In these fearlearning paradigms, $A h i 1^{+/-}$exhibited a diminished ability to memorize the association between the shock and the context (b) or cue (c). Although baseline freezing (percentage of time freezing during $30 \mathrm{~s}$ ) was similar across genotypes (habituation and pretone periods), as was the magnitude of freezing increment in the immediate postshock period (b), during the test, exposure to the preconditioned context (b) or cue (tone, c) resulted in freezing responses that were significantly lower in the Ahi ${ }^{+/-}$mice compared with $A$ hi $1^{+/+}$littermates, leading to a significant conditioning state vs genotype interaction. ${ }^{* * *} P<0.001$ compared with the same genotype's freezing during the prior conditioning state. ${ }^{++} P<0.01$ compared with $A h i 1^{+/+}$mice exposed to the conditioned stimulus. $n=15$ mice/group. Data are expressed as mean \pm s.e.m. 
difference was only evident during the tone (independent samples test, $t_{19.9}=3.5, P=0.002$ ).

Relationship between Ahi1 expression and fear learning across the BXD RI strains

Given the distinct phenotypes observed in $\mathrm{Ahi1}^{+/-}$mice with respect to fear learning, we attempted to correlate these traits with physiological variation in neocortical Ahi1 expression, measured in neonates (P3), in juveniles (P14) and in adults, across the BXD RI strains. In both developmental neocortical expression data sets (BIDMC/UTHSC Dev Neocortex P3 and BIDMC/UTHSC Dev Neocortex P14) and in the corresponding adult data set (HQF BXD Neocortex P60), we identified two probes for Ahi1, one located in exon 5 and the other in the gene's $3^{\prime} U T R$. The latter probe was chosen for further analysis owing to its consistently higher expression (Supplementary Table S1). Expression profiles measured at each of the three developmental stages were normally distributed, yet highly variable, across strains (Figures $3 a$ and b, Supplementary Figure S1a and Supplementary Table S2).
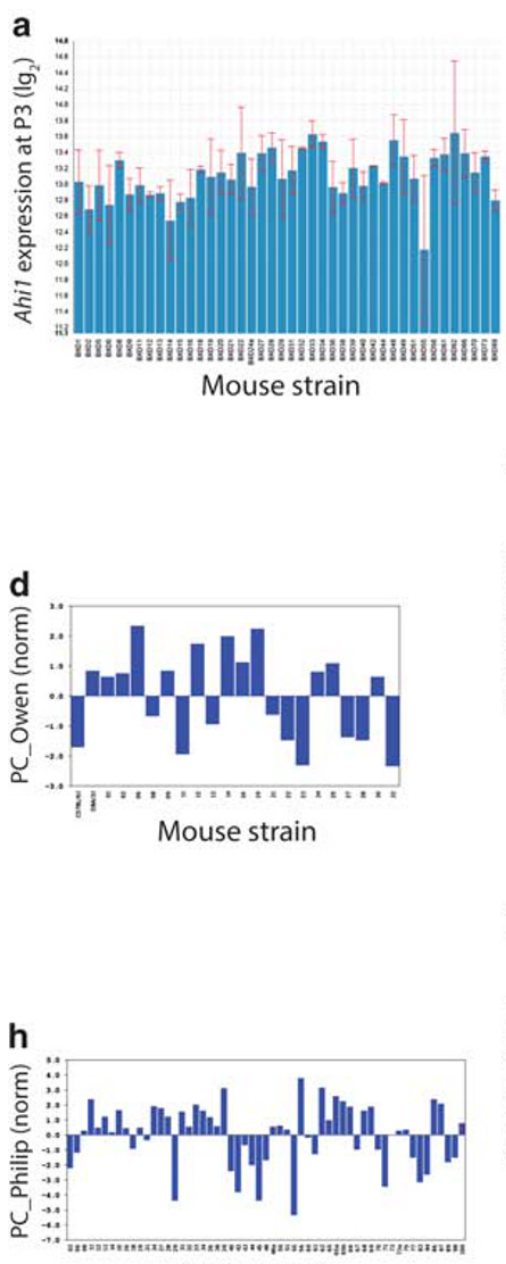

Mouse strain

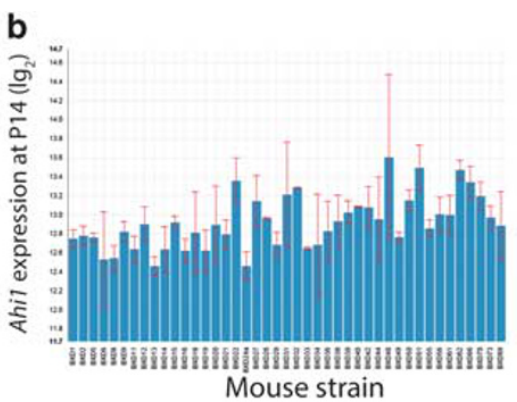

f
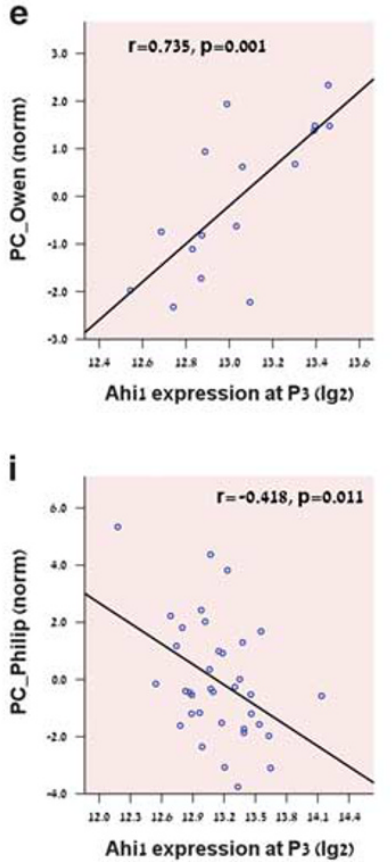
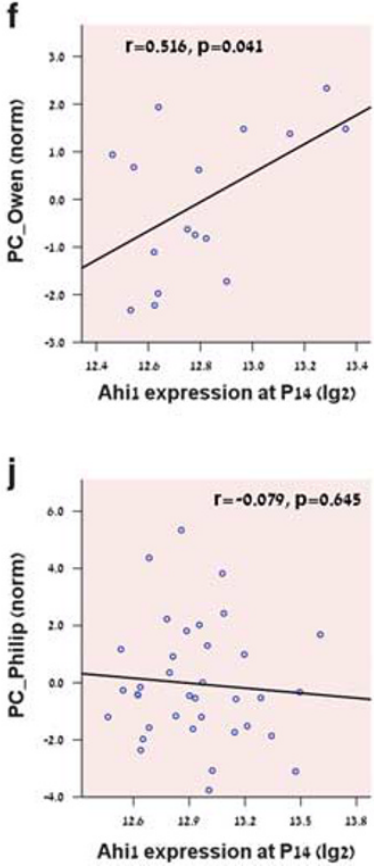
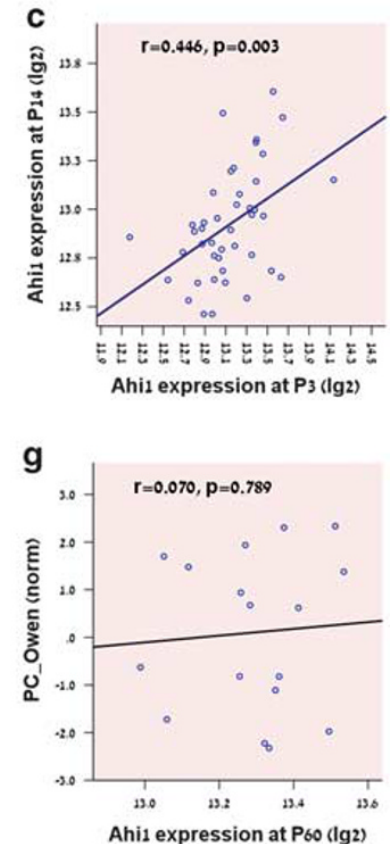

Ahi1 expression at $P_{3}\left(\lg _{2}\right)$

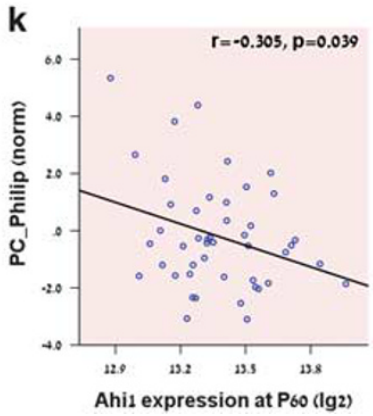

Figure 3. Relationship between Ahi1 expression and fear learning across the BXD recombinant inbred strains. (a, b) Bar graphs depicting the expression of the Ahi1 3'UTR (untranslated region) probe (y axis, arbitrary units $\left(\log _{2}\right)$ ) across the BXD RI and parent strains assessed in the neocortex at postnatal days (a) P3 and (b) P14. Note variability in probe expression across strains (2-3-fold range). (c) Pearson's productmoment correlation between expression of the Ahi1 3'UTR probe measured at P14 (y axis) vs P3 ( $x$ axis). Note that, although significant, it is only modest in strength. (d) Bar graph depicting 'PC_Owen', the major principal component (PC) derived from three traits related to contextual fear conditioning reported by Owen et al. ${ }^{53}$ across adult mice from $23 \mathrm{RI}$ strains. Note that PC trait is highly variable across strains. $(\mathbf{e}-\mathbf{g})$ Pearson's product-moment correlations between 'PC_Owen' ( $y$ axis) and expression of the Ahi1 3'UTR probes ( $x$ axis) measured at (e) P3, (f) P14 and (g) P60. Only strains for which both expression and behavioral data were available were entered into the correlation. Note the strong, highly significant correlation between contextual fear learning in adulthood and Ahi1 expression in neonates. Fear learning was not correlated with Ahi1 expression in adulthood and only marginally correlated with Ahi1 expression in juveniles. (h) Bar graph depicting 'PC_Philip', the major PC derived from six traits related to contextual and cued fear conditioning reported by Philip et al. ${ }^{54}$ across adult mice from 60 Rl strains. (i-k) Pearson's product-moment correlations between 'PC_Philip' ( $y$ axis) and expression of the Ahi1 3'UTR probes ( $x$ axis) measured at (i) P3, (j) P14 and (k) P60. Note that cued fear learning in adult mice was moderately correlated with the neonatal expression profile of Ahi1 and weakly correlated with the adult profile. Notice that although the correlations between Ahi1 expression and PC_Philip were negative, the traits reported by Philip et al. ${ }^{54}$ reflect activity upon presentation of the conditioned stimulus, while those reported by Owen et al. ${ }^{53}$ reflect freezing. Hence, correlations between Ahi1 expression and fear conditioning in these two reports point to the same biological direction, so that strains exhibiting lower neocortical Ahi1 expression tend to perform worse on these associative learning paradigms. The data in the bar graphs depict mean \pm s.e.m. calculated over samples from each individual RI strain. For sample sizes, please refer to individual reports. ${ }^{53,54}$ 
Moreover, while expression profiles at P3 and P14 were modestly correlated across strains ( $r_{\text {pearson }}=0.446, P=0.002$, Figure $3 c$ ), they were weakly correlated with the P60 expression profile (Supplementary Figures $1 \mathrm{~b}$ and $\mathrm{c}$ ), suggesting that (i) significant changes in Ahi1 expression occur upon maturation of the brain and (ii) these changes themselves are variable across strains.

Using a predefined keyword search across the BXD Published Phenotypes Database, we identified four independent scientific reports measuring contextual and cued fear conditioning ${ }^{53-56}$ (Supplementary Tables S3 and S4). Principal component (PC) analysis revealed single PCs accounting for $>65 \%$ of the variability in the traits reported by Owen et al. ${ }^{53}$ (PC_Owen derived from three traits related to contextual conditioning, Figure $3 \mathrm{~d}$ and Supplementary Table S5), by Philip et al. ${ }^{54}$ (PC_Philip derived from six traits related to contextual and cued conditioning, Figure $3 \mathrm{~h}$ and Supplementary Table S6) and by Brigman et al. ${ }^{55}$ (PC_ Brigman derived from three traits related to contextual and cued conditioning, Supplementary Table S7). There was only one relevant trait reported by Yang et al. ${ }^{56}$ (10901_Yang). PC_Philip and PC_Brigman were the only significantly intercorrelated PCs (Supplementary Table S8), suggesting considerable intra-strain differences across the four reports.

Three of the abovementioned reports recorded data on at least 10 strains that were also represented in the relevant neocortical expression data sets. ${ }^{53-56}$ Although a strong, highly significant correlation across strains was noted between PC_Owen and the P3 Ahi1 expression profile $\left(r_{\text {pearson }}=0.735, \quad P_{\text {raw }}=0.001\right.$, $\left.P_{\text {adj }}=0.009\right), P C \_O w e n ' s$ correlation with the P14 profile, which was only moderate, did not withstand correction for multiple testing $\left(r_{\text {pearson }}=0.516, P_{\text {raw }}=0.041, P_{\text {adj }}=0.092\right.$, Figures $3 e$ and $f$, respectively). PC_Owen and the P60 Ahi1 expression profile were not correlated at all $\left(r_{\text {pearson }}=0.070, P_{\text {raw }}=0.790\right.$, Figure $3 g$ ). With respect to PC_Philip, a significant correlation across strains with the P3 Ahi1 expression profile was noted $\left(r_{\text {pearson }}=-0.418\right.$, $P_{\text {raw }}=0.011, P_{\text {adj }}=0.0495$, Figure 3i). PC_Philip's correlations with the P14 and P60 profiles were non-significant $\left(r_{\text {pearson }}=-0.305\right.$, $P_{\text {raw }}=0.039, \quad P_{\text {adj }}=0.092$ and $r_{\text {pearson }}=-0.079, \quad P_{\text {raw }}=0.645$, Figures $3 \mathrm{j}$ and $\mathrm{k}$, respectively). A detailed summary of Pearson's product-moment and Spearman rank-order correlations between the Ahi1 expression profiles and fear conditioning traits is provided in Supplementary Tables S9 and S10. Notably, although the correlations between Ahi1 expression and PC Philip were negative, the traits reported by Philip et al. ${ }^{54}$ depict activity upon presentation of the conditioned stimulus, while those reported by Owen et al. ${ }^{53}$ relate to freezing. Hence, correlations between Ahi1 expression and fear conditioning in these two reports point essentially to the same biological direction, so that strains exhibiting lower neocortical Ahi1 expression tend to perform worse on these associative learning paradigms. Finally, hierarchical regression analysis revealed that while Ahi1 expression at P3 predicted $48 \%$ and $23 \%$ of the total variance in PC_Owen and PC_Philip, respectively, addition of the P14 and P60 time points to the earlier one did not improve model strength (Supplementary Tables S11 and S12). These data suggest that Ahi1 expression level during early neurodevelopment may be a critical determinant of fear-learning performance in adulthood.

QTLs modulating Ahi1 expression and fear learning across the BXD RI strains

Given the implied role of Ahi1 expression levels during early neurodevelopment in determining associative learning in adulthood, we next performed an eQTL analysis of Ahi1, as measured within the $\mathrm{P} 3$ neocortical expression data set, by computing linkage maps across the entire genome. A trans-eQTL with a maximum logarithm of odds score of 4.59 , well above the wholegenome significance cutoff of 3.80, was mapped to Chr1:
69.048453, (Figures 4a-c and Supplementary Figure S3). This locus harbors an intronic single-nucleotide polymorphism (rs6312657) within the ErbB4 gene that varies across the BXD progeny. The DD genotype was associated with mean Ahi1 levels that were roughly $\sim 35 \%$ higher compared with the BB genotype $\left(t_{41}=5.1, P<0.0005\right.$, Figure 4d). The PC_Owen, PC_Philip and PC_Brigman traits included strain-specific data that partially overlapped the rs6312657-genotyped data set, so that at least eight phenotyped mice could be grouped into each homozygous genotype category (Supplementary Table S13). Notably, out of these PC traits, rs6312657 polymorphisms were associated with significant differences in PC_Brigman, so that strains carrying the DD genotype displayed enhanced fear learning compared with BB carriers $\left(t_{18}=2.687, P_{\text {raw }}=0.015, P_{\text {adj }}=0.045\right.$, Figure $4 \mathrm{e}$ and Supplementary Table S14).

Next, we sought to determine whether expression of other relevant genes during neocortical development is also modulated by the same locus that regulates Ahi1 expression. eQTL analysis of the entire BIDMC/UTHSC Dev Neocortex P3 data set revealed 41 transcripts that have a single best eQTL (trans-eQTL in all cases) that can be mapped to the Chr1: 69.048453 locus with logarithm of odds scores $>3.80$ (Supplementary Table S15). Among those 41 transcripts, enrichment analysis revealed significant overrepresentation of a subset of seven genes involved in key biological processes such as neuron development (GO:0048666, $\left.P_{\text {raw }}=5.38 \times 10^{-11}, \quad P_{\text {adj }}=1.38 \times 10^{-8}\right)$, neuron differentiation (GO:0030182, $\quad P_{\text {raw }}=2.95 \times 10^{-10}, \quad P_{\text {adj }}=2.53 \times 10^{-8}$ ) and cell projection organization (GO:0030030, $P_{\text {raw }}=2.22 \times 10^{-10}$, $P_{\text {adj }}=2.53 \times 10^{-8} \quad$ (Figure $4 \mathrm{f}$ and Supplementary Table S16). Notably, besides Ahi1, coded by these seven genes are proteins such as Hap1, which forms, together with Ahi1, a stable protein complex that is critical during early brain development, and may be involved in emotional regulation. ${ }^{57-59}$ Finally, using a heat map visualization that facilitates detection of common QTLs for sets of traits, sharing of suggestive and significant QTLs, other than at Chr1: 69.048453, was observed (i) among the subset of seven genes; and (ii) between these genes and the fear conditioning traits (Figure $4 \mathrm{~g}$ ).

\section{Studies of functional brain networks}

Given the recently proposed placement of brain connectivity as an 'intermediate phenotype' positioned between genetics and behavior, ${ }^{6}$ together with the long-lasting effects that naturally occurring or genetically manipulated variations in Ahi1 expression exert in terms of cognitive-emotional outcomes, we next attempted to determine the effects of reduced Ahi1 expression, evident in the Ahi1 knockout mouse, on the topology of wholebrain functional networks. The nodes depicted in these networks were based on 27 preselected ROls (Supplementary Table S17).

Small-world organization of resting-state brain networks. The small-world index of a network is defined as the ratio of the global clustering coefficient to the mean shortest path length, after both metrics have been standardized by comparing their values to those in equivalent random networks. ${ }^{4}$ Networks were evaluated across connection densities greater than the minimal density of 0.34 , at which all nodes were connected for the first time. Whole-brain networks of both $\mathrm{Ahil}^{+/+}$and $\mathrm{Ahi1}^{+/-}$mice had clustering coefficients that were higher than those expected in random networks, while having shortest path lengths similar to random networks. This pattern resulted in small-world indices greater than one across densities (Supplementary Figures S4a-c). However, between-genotype differences in these global network metrics were non-significant (Supplementary Figures S4d-f). As expected, when the connection density was increased, the smallworld index $\sigma$ monotonically declined toward an asymptotic value of 1 . For densities $>0.50$, we found that $\sigma \leqslant 1.2$, indicating that the 

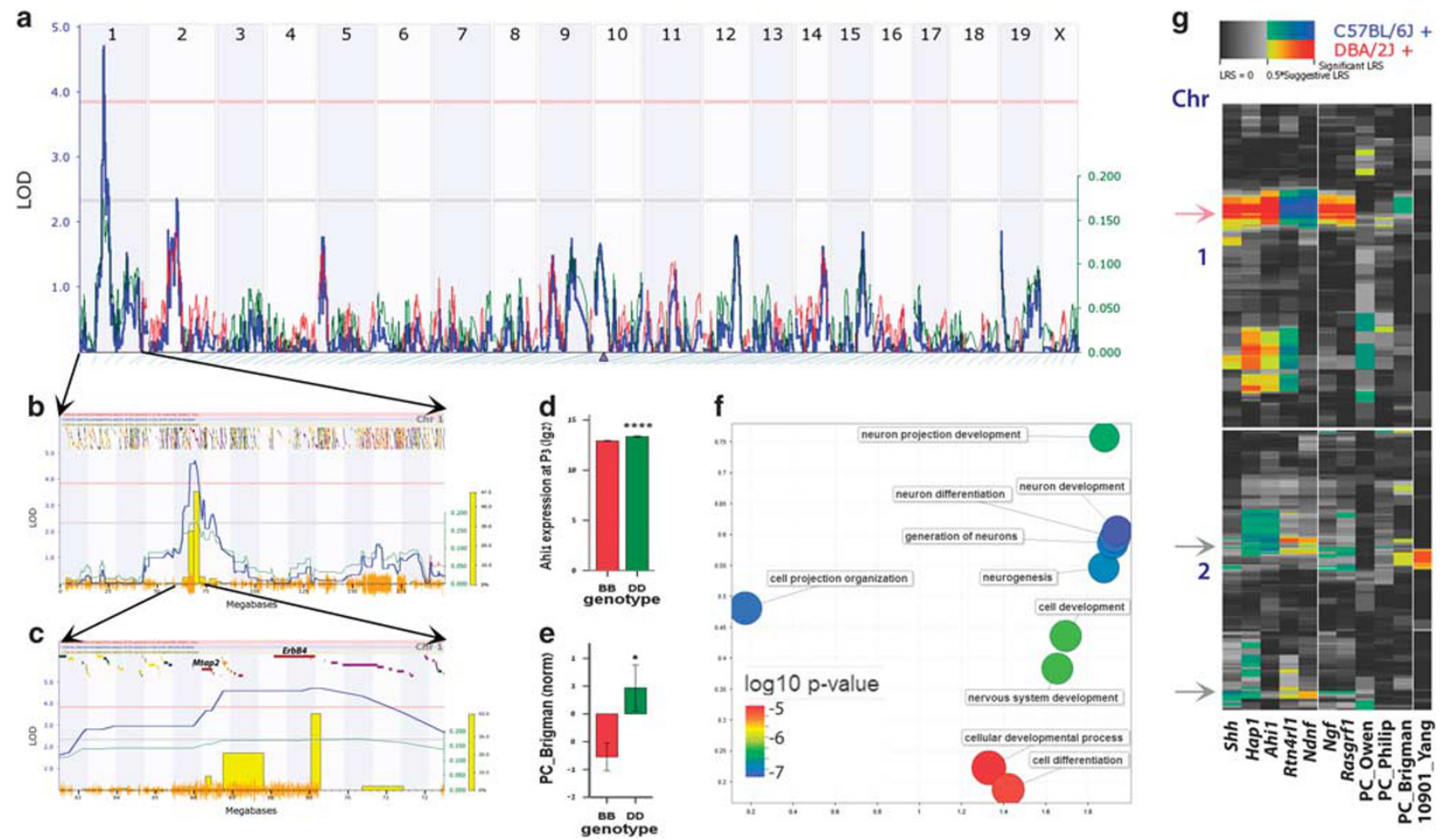

Figure 4. Mapping quantitative trait loci (QTLs) that modulate Ahi1 expression and fear conditioning. (a-c) The major QTL controlling the expression of Ahi1 in the neocortex during early neonatal life, identified by interval mapping the Ahi1 probe (ILMN_2813712) from the BIDMC/ UTHSC Dev Neocortex P3 database against dense genotype data for 43 BXD RI strains. Plots show interval maps for the whole genome (a), for Chr 1 (b) and for the maximal QTL region on chromosome 1 at 66-72 Mb (c). The $x$ axis represents the physical map of the chromosome(s); the $y$ axis and thick blue line provide the logarithm of odds (LOD) score of the association between Ahi1 expression and the genotypes of markers. The two horizontal lines are the suggestive (gray) and significance (pink) thresholds computed using 5000 permutations. The secondary lines indicate whether C57BL/6J (red) or DBA/2J (green) alleles increase Ahi1 expression values. The additive effect of these alleles is quantified using the green scale on the far right of the plot, which represents normalized $\log _{2}$ expression values. Yellow bars (in panels $\mathbf{b}$ and $\mathbf{c}$ ) indicate results of a bootstrap analysis evaluating approximate confidence limits of QTL peaks. Orange lines show single-nucleotide polymorphism density. Color bars above the thick blue line denote actual gene locations. Note position of the ErbB4 and Mtap2 genes. (d, e) Effect plots of marker rs6312657 (Chr 1 at $69.048453 \mathrm{Mb}$ ) on the expression of Ahi1 in the neocortex during early neonatal life (d) and on fear conditioning during adulthood (PC_Brigman, e). Notice higher phenotypic means for the D allele when compared with the $B$ allele. This difference is significant and therefore results in the presence of a single QTL modulating both traits. ${ }^{*} P<0.05,{ }^{* * * *} P<0.0005$. (f) Enrichment analysis based on GO terms among 41 transcripts that have a single best trans-eQTL that can be mapped to the Chr1: 69.048453 locus with LOD scores $>3.80$. Only biological processes are depicted. Bubbles are color-coded according to the false-discovery rate-adjusted log 10 -value of category-enriched genes relative to the whole mouse genome. Significance ranges from $\sim 2 \times 10^{-8}$ (blue) to $\sim 2 \times 10^{-5}$ (red). The $x$ and $y$ coordinates depict semantic similarities among GO terms. (g) QTL 'heat map' of LOD scores, with red indicating modulation by DBA/2J alleles and blue indicating modulation by $\mathrm{C} 57 \mathrm{BL} / 6 \mathrm{~J}$ alleles. More intense colors mark chromosomal regions with comparatively high linkage statistics. Only loci with LOD scores $>(0.5 \times$ suggestive threshold $)$ are color-coded. The individual traits are run side by side to enable easy detection of common and unique QTLs. Seven transcripts involved in neuronal development, differentiation and projections and four fearconditioning traits (Supplementary Tables S16 and S8, respectively) are displayed. The genome location is shown along the long axis of the map, marker by marker. As it is intended for qualitative assessment, only two representative chromosomes (1 and 2$)$ are depicted. Notice that sharing of QTLs among the subset of seven transcripts and between them and the fear conditioning traits is evident not only at the significant Chr1: 69.048453 QTL (pink arrow) but also at several other suggestive (or near-suggestive) QTLs (gray arrows).

brain networks were becoming topologically indistinguishable from random graphs. Thus we identified the range of densities $0.34 \leq d \leq 0.50$ as the regime associated with small-world properties of fully connected functional networks.

Assortative organization of resting-state brain networks. The assortativity coefficient $(a)$ is the Pearson correlation coefficient of degree between pairs of linked nodes ${ }^{46}$ (Supplementary Figures S5a and b). Quantified for each individual functional network, $a$ was distributed normally across mice (Supplementary Table S18). At minimum network density $(d=0.34)$, a ranged between 0.01 and 0.56 . Notably, although the total number of edges (that is, connections) is identical when networks are held at same densities, individual differences in assortativity produced strikingly different topologies. Although these differences are most prominent graphically when nodes were positioned according to their degree metrics and edges were binarized (Figures 5a and $b$ ), anatomical and heat map visualizations of these same networks, in which edges depict the weighted strength of the functional connections, convey additional information. In the individual networks presented, the non-assortative network displays strong intra-cortical connections, with moderate longrange functional connections between rostral and caudal structures (Figures $5 \mathrm{c}$ and e). On the other hand, while the assortative network displays dense mesolimbic connections, long-range rostrocaudal connections are sparse (Figures $5 \mathrm{~d}$ and f). Beyond 
qualitative examination of individual networks with different assortativity coefficients, graph-based analysis can provide valuable quantitative information that enables direct between-group comparisons. On a group level, $\mathrm{Ahil}^{+/-}$mice displayed an assortative mixing pattern whereas the topology of the $A h i 1^{+/+}$ mice was virtually non-assortative (Figure $5 \mathrm{~g}$ ). This difference remained significant across densities (bootstrap for independent samples test, $P=0.016-0.058, P_{\text {AUC }}=0.026$, Figure $5 \mathrm{~h}$ ).

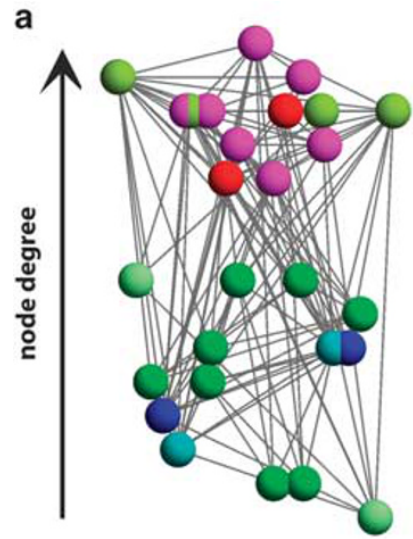

C
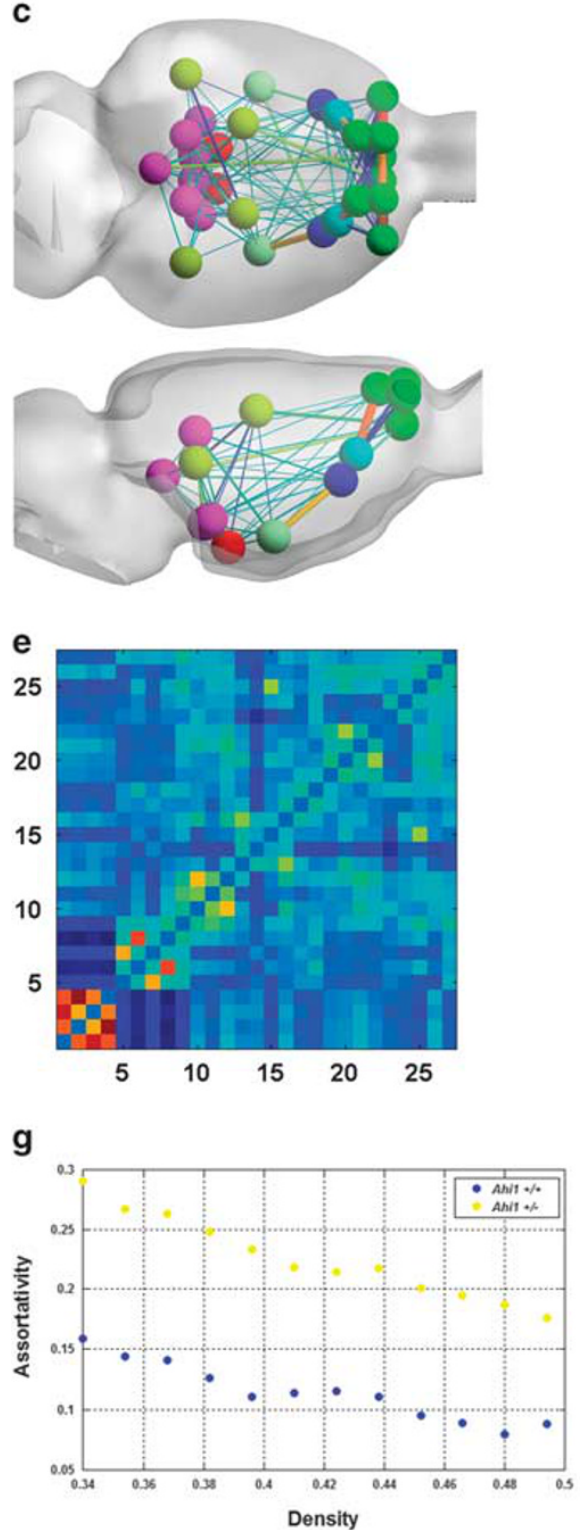

b

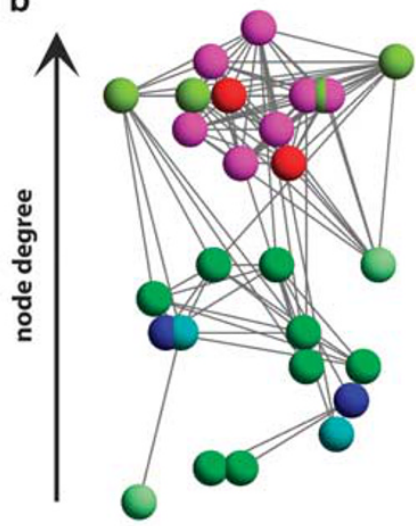

d
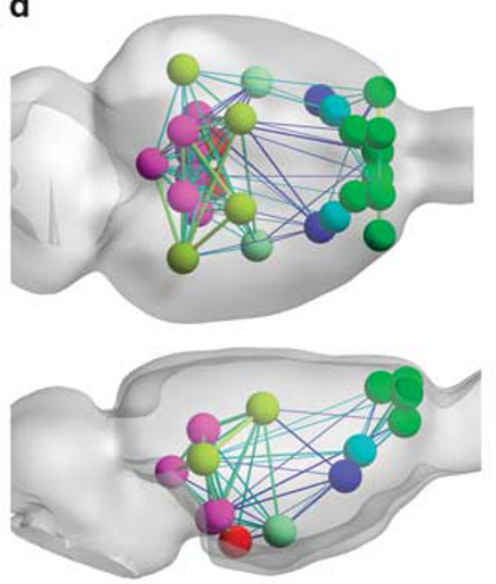

f
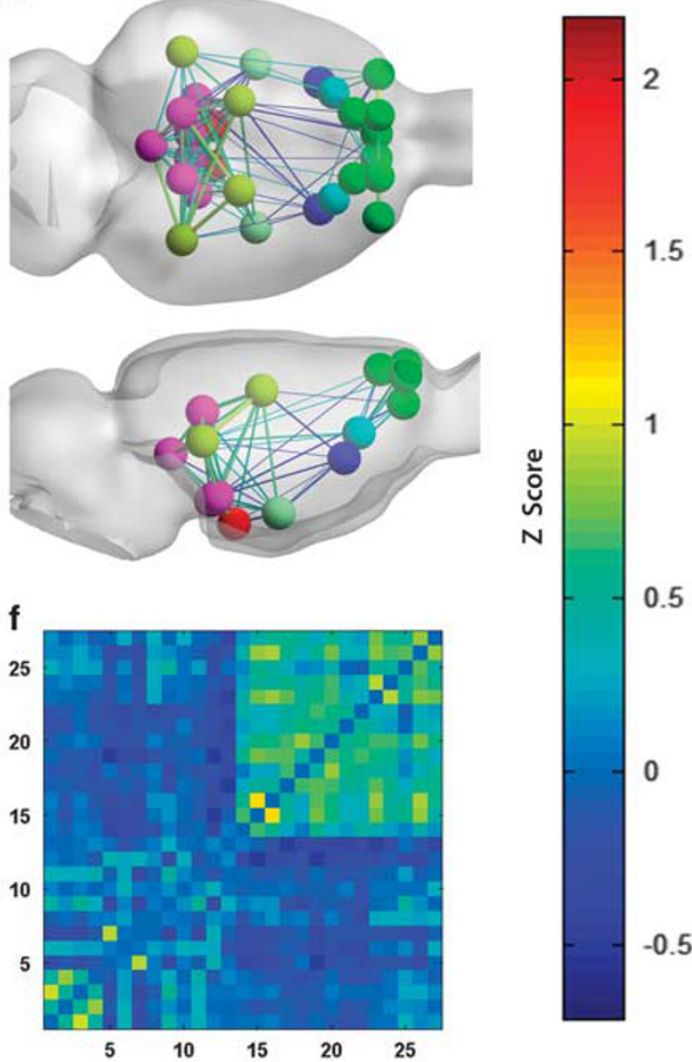

h

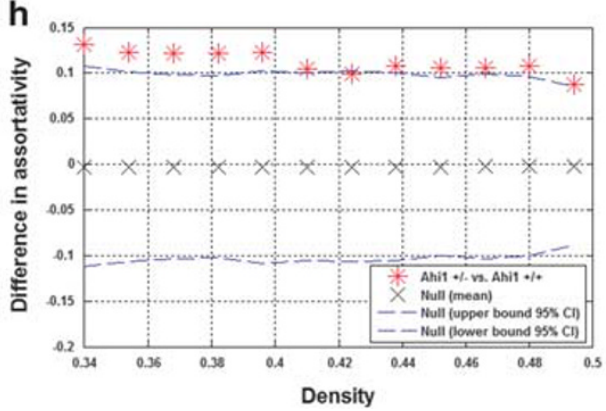

Figure 5. For caption please refer page 892. 
Hierarchical organization of resting-state brain networks. The hierarchy coefficient $(\beta)$ depicts the power-law relationship between each node's local clustering and degree metrics across the entire network ${ }^{47}$ (Supplementary Figure S5c). Quantified per each individual functional network, hierarchy was inversely correlated with assortativity across mice $(r=-0.695, P<0.001$, Supplementary Figure S5d). At minimum network density $(d=0.34), \beta$ ranged between -0.45 and 0.42 . Again, while these differences are most conspicuous when presented in graph format (Figures $6 a$ and b), anatomical and heat map visualizations of these same networks revealed that compared with the dis-hierarchical one, the hierarchical network displays strong local intra-cortical connections with prominent corticolimbic and mesocortical anticorrelations (Figures $6 c-f$ ). Beyond quantification of individual network hierarchies (Figures $6 \mathrm{~g}$ and $\mathrm{h}$ ), graph-based group estimates revealed that while $A h i 1^{+/+}$mice displayed a hierarchical network topology (mean $\beta=0.16,95 \%$ confidence interval (Cl) $0.07-0.25$, their $A h i 1^{+/-}$littermates displayed a non-hierarchical pattern (mean $\beta=-0.06,95 \% \mathrm{Cl}-0.19$ to 0.09 ). The resulting between-group difference was significant when hierarchy was measured in networks held at minimum density (bootstrap for independent samples test, $P=0.022$ ) as well as in networks derived from parameter area under the curve-averaged across densities (same test, $P=0.019$ ).

Regional functional connectivity of the SC. Important brain regions (hubs) often interact with many other regions, facilitate functional integration and have a key role in network resilience to insult. Measures of node centrality variously assess importance of individual nodes on the above criteria. Nodal degree is one of the most common measures of centrality and has a straightforward neurobiological interpretation: nodes with a high degree are interacting, structurally or functionally, with many other nodes in the network. ${ }^{5}$ As the superior colliculi (SC) serve as relative hubs both in the non-assortative as well as in the assortative network (pink nodes in Figure 5), and given their unique roles in the processing of sensory information, ${ }^{60}$ we next attempted to compare differences in the SC's nodal degree between wild-type mice and $\mathrm{Ahi1}^{+/-}$littermates. Using a one-way multivariate analysis of variance with the right and left SC as the dependent variables, we detected a statistically significant difference between genotypes on the combined end point $\left(F_{[2,24]}=3.514, P=0.046\right.$; Wilks' $\Lambda=0.774$; partial $\eta^{2}=0.226$ ), suggesting that the SC could serve as an example for the association between Ahi1 underexpression and distinct spatial patterns of functional connectivity.

\section{DISCUSSION}

We have reported a composite effort to link early variations in the expression of the neurodevelopmental gene Ahi1 to long-term effects on functional brain networks and cognitive-emotional phenotypes. Our behavioral findings suggest that under test conditions which bring forth their stress-resilient phenotype (swim-to-visible-platform) but do not present a significant

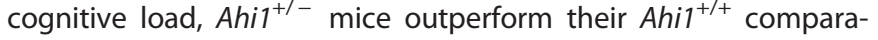
tors. However, under test conditions that obviate betweengenotype stress-related differences, as is the case with the NORT (minimal stress) and fear conditioning paradigms (bypass stress resilience), $A h i 1^{+/-}$mice display consistent cognitive deficits. These findings were corroborated by quantifying neonatal expression $\times$ adult behavior correlations and neonatal eQTL $\times$ adult QTL relationships, using a comprehensive neuroinformatic database of Rl mouse strains, in which Ahi1 expression is measured as a continuum at several developmental points. Finally, using resting-state $\mathrm{fMRI}$ we demonstrated that whole-brain functional networks in $\mathrm{Ahi1}^{+/-}$mice display strikingly different properties in terms of their assortative and hierarchical organization compared with $\mathrm{Ahi1}^{+/+}$littermates.

Introducing rodents into a novel environment causes a significant stress response. ${ }^{61}$ In such situations, rodents are often inclined to mainly explore the peripheral zones. The tendency to remain near the walls, thigmotaxis, serves as a reliable ethological index of anxiety. ${ }^{62}$ In a new cohort of young adult mice, we have replicated our previous finding regarding the anxiolytic-like phenotype manifested by $\mathrm{Ahi1}^{+/-}$mice under neophobic conditions. ${ }^{31}$ Imposing forced activity, such as forced swimming, likewise induces a profound stress response in rodents. ${ }^{63}$ Not surprisingly, when faced for the first time with the combination of forced swimming and a novel environment, such as in the MWM, mice display strongly thigmotactic behavior. Thigmotaxis and anxiety have been negatively correlated with performance in this scenario. For instance, relative to males, naive female rats displayed higher anxiety and inferior performance in swimming to a visible platform of a MWM owing to strongly thigmotactic swimming. ${ }^{36}$ Hence, it is plausible that the $A h i 1^{+1-}$ mice outperformed their $\mathrm{Ahi1}^{+/+}$controls in this straightforward, learningindependent task by virtue of their relative stress resilience.

Rodents have a tendency to interact more with a novel object than with a familiar object. This tendency has been used to study learning and memory. ${ }^{64}$ In fact, the NORT is not only very sensitive and capable of detecting subtle differences in memory (discrimination) but also in exploratory performance. ${ }^{65}$ As a consequence, it is susceptible to potential biases owing to neophobic stress. By choosing a small apparatus with which the

Figure 5. Assortative organization of whole-brain functional networks. (a-f) Examples of functional networks derived from individual mice held at minimum density $(d=0.34)$. ( $\mathbf{a}, \mathbf{b})$ Schematic visualizations of non-assortative $(\mathbf{a}, a=0.01)$ and highly assortative $(\mathbf{b}, a=0.56)$ networks. Node position along the $y$ axis corresponds to nodal degree. Edges are binarized. Note that, while in the assortative network nodes tended to preferentially connect to other nodes with a similar degree, connections in the non-assortative network seem random in that sense. Moreover, although the total number of edges is identical in both networks, the difference in assortativity gives rise to strikingly different topologies. (c, d) Anatomical visualizations of these same networks, viewed from the horizontal and sagittal planes (top and bottom images, respectively). These images were produced with weighted edges, which depict the strength of the functional connections. Edge colors correspond to the Fisher-transformed Z-score of the correlation coefficient between each pair of connected nodes (see color bar to the right). Only the top $34 \%$ of connections, in terms of their absolute Z-score, are depicted. Nodes are color-coded according to the different brain regions that they represent: Dark green, cortical regions; light blue, striatum; marine blue, putamen; light green, amygdala; olive, hippocampus; pink, midbrain structures; red, mammillary bodies. (e, f) Heat maps of the adjacency matrices derived from these same networks, using the same Z-score color bar as above. Regions of interest are presented according to their anatomical position along the rostrocaudal axis, so that the most rostral are assigned numbers 1 and 2 and the most caudal 26 and 27. (g) Changes in network assortativity as a function of network density. Mean network assortativity for $\mathrm{Ahil}^{+/+}$and $\mathrm{Ahi}^{+/-}$mice (blue and yellow, respectively) are depicted across densities within the small-world regime. Note that $\mathrm{Ahi}^{+/-}$mice displayed an assortative pattern across densities, compared with a non-assortative topology displayed by $\mathrm{Ahi}^{+/+}$mice. (f) Between-genotype differences in network assortativity as a function of network density. The $95 \%$ confidence intervals and between-group differences in assortativity are depicted. The red ${ }^{*}$ marker shows the difference between $A$ hi $1^{+/-}$and $A h i 1^{+/+}$networks; thus ${ }^{*}$ signs falling above the confidence intervals indicate densities in which $A h i 1^{+/-}$mean network assortativity is significantly higher than $A h i 1^{+/+}$. Relative to the $A h i 1^{+/+}$networks, the assortativity coefficients of the $A h i 1^{+/-}$networks were significantly higher across almost all densities. $n=13\left(\right.$ Ahi $\left.1^{+/+}\right)$, $n=12\left(\right.$ Ahi1 $\left.^{+/-}\right)$. 
a

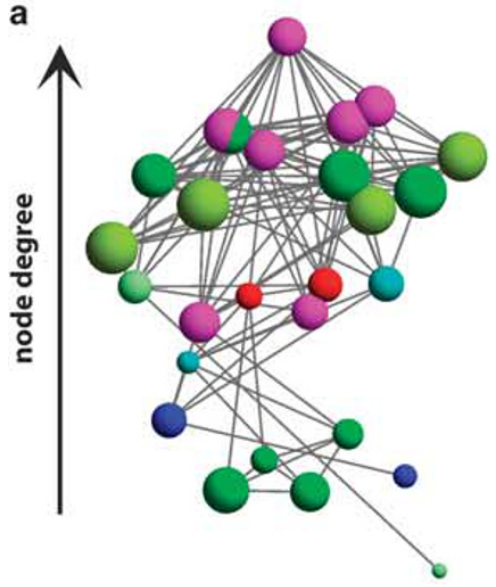

C
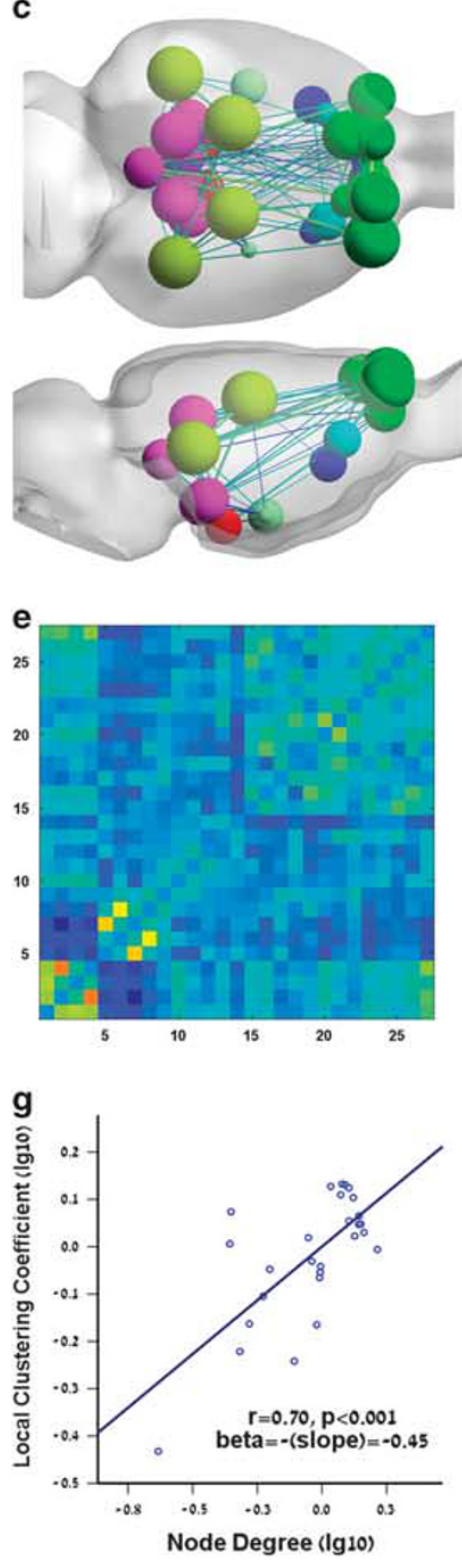

b

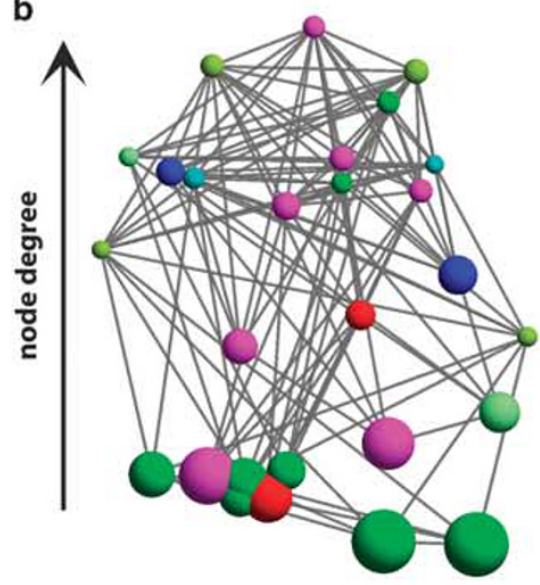

d
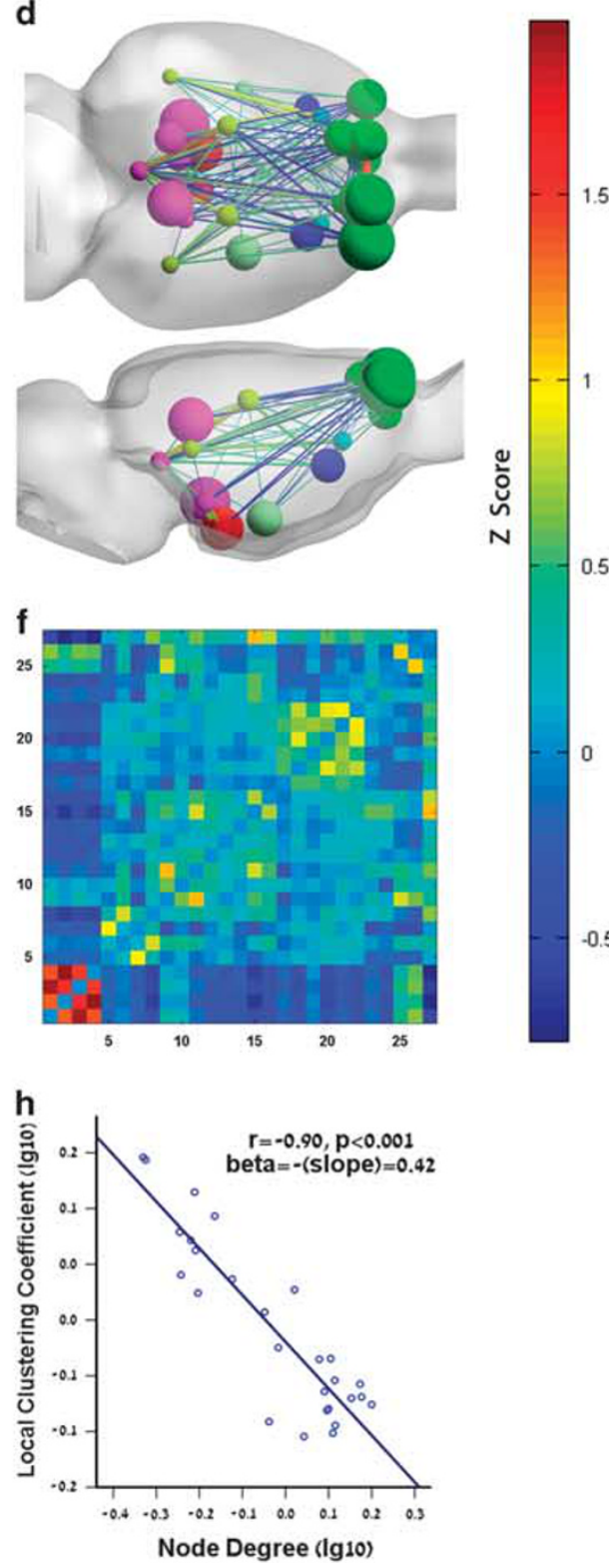

Figure 6. For caption please refer page 894. 
animals have been familiarized with during pretraining, the OF situation that evokes anxiety and stress-related behaviors such as thigmotaxis can be minimized. ${ }^{50}$ Thus the minimal amount of exploration required for stable discrimination performance is achieved. ${ }^{65}$ Under such circumstances, it is plausible that a genetically determined 'advantage' of a knockout mouse, in terms of stress resilience and exploratory performance, could be minimized. In this task, Ahi1 $1^{+/+}$mice outperformed their $\mathrm{Ahi1}^{+/-}$ littermates. The superior performance of $\mathrm{Ahi}^{+/+}$mice in the NORT, as opposed to their inferior performance in the MWM, may thus be due to fundamental differences in these two tasks in terms of the skills required (learning vs exploration and agility) and the stress imposed (low vs high).

As opposed to the above-mentioned paradigms, in which performance can be interpreted in a relatively straight-forward manner, deciphering what underlies the marked reduction of fear memory retrieval in $\mathrm{Ahi1}^{+/-}$mice in the context and cued fear conditioning paradigms is more challenging. The immediate footshock-induced freezing response serves as an innate defense mechanism when animals are presented with a sudden nociceptive stimulus and has been used as a model for assessing anxiolytic drugs. ${ }^{66}$ Thus our results showing similar betweengenotype freezing in the immediate postshock period of the training phase suggest that an ability to experience anxiety is preserved in $\mathrm{Ahi1}^{+/-}$mice. Notably, it was recently suggested that this immediate response is primarily elicited through the spinoparabrachio-amygdaloid pathway. ${ }^{67}$ In this way, pain could elicit direct amygdalar activation, obviating the need for intact corticolimbic circuitry. To this end, we have previously demonstrated that corticolimbic disconnectivity could underlie the stressresilient phenotype evident when $\mathrm{Ahil}^{+/-}$mice are exposed to environmental threats. ${ }^{31}$ We have also shown that pentylenetetrazole and caffeine, which are presumed to induce direct activation of the amygdala, brought about similar increases in serum corticosterone in both genotypes. Thus it seems reasonable to assume that when cortical deficits in threat detection are bypassed, through either nociception or pharmacological manipulation, $A h i 1^{+/-}$mice retain an inherent ability to 'feel' anxious.

In contrast to immediate foot-shock-induced freezing, fear conditioning involves the pairing of a neutral stimulus with an aversive, unconditioned stimulus (US). ${ }^{52}$ The neutral stimulus initially elicits no emotional reaction, but after repeated pairings with the US, the neutral stimulus becomes a conditioned stimulus signaling imminent US onset and inducing anxiety associated with the anticipation of the aversive US. Fear conditioning is generally an adaptive and self-preserving form of associative learning. As such, it is recognized as a robust model system to investigate the neurobiological mechanisms of learning and memory in the mammalian brain. Although responding to the US largely depends on ascending spinal pathways, learning to associate it with the conditioned stimulus requires the participation of additional circuits, including the hippocampus and entorhinal, perirhinal and cingulate cortices. In fact, based on c-Fos immunoreactivity, the degree of freezing to conditioned context and tone has recently been correlated with activation of the entorhinal cortex, hippocampus (granule cell layer) and lateral amygdala. ${ }^{68}$

Given the previously demonstrated functional disconnectivity between the amygdala, ventral hippocampus and entorhinal cortex that characterizes the $A h i 1^{+/-}$mice, our fear conditioning results could be viewed as a cognitive deficit attributed to such disconnectivity. This view is supported by the underperformance of $\mathrm{Ahi}^{+/-}$mice in the NORT, as the most essential brain structures implicated in this cognitive task are also the hippocampus and rhinal cortices. ${ }^{69}$ Notably, in a recent study of mice with deletion of Rapgef6, another schizophrenia-related gene, the authors also attributed an anxiolytic-like phenotype, along with deficits in fear conditioning, to impaired hippocampal and amygdalar function. ${ }^{70}$ Although in our case this interpretation is well justified, others are plausible. Fear conditioning has long been implicated in the pathogenesis of stress-related psychopathology. ${ }^{71}$ In fact, higher conditionability has been associated with development of pathological anxiety. ${ }^{72}$ In this sense, the decreased conditioning response of the $\mathrm{Ahi}^{+/-}$mice could (also) be viewed as a form of resilience.

Across mammals, Ahi1 expression is critical for normal cortical development. In humans, homozygous AHI1 mutations have been shown to impair neuronal migration, giving rise to aberrant cortical architecture. $^{73}$ In mice, loss of Ahi1 affected early development by impairing neuronal differentiation ${ }^{22}$ and cortical axonal decussation. ${ }^{74}$ As major cortical inputs are implicated in associative learning, it is plausible to assume that physiological variation in Ahi1 expression during neurodevelopment exerts long-lasting effects on the efficiency of this cognitive domain. To this end, the findings obtained from the neuroinformatic analysis, suggesting significant correlations across the BXD RI strains between neocortical Ahi1 expression and fear conditioning to both context and cue, provide independent support for the data obtained from our Ahi1 knockout model. ${ }^{75}$

This neuroinformatic analysis also supports further insights. Our knockout mouse, much like other genetic models, provides a dichotomous system for exploring the effects of a gene's altered expression compared with its 'natural' expression. Although utilization of such models may improve chances to reveal a given gene's role, genetic variations that cause more subtle alterations of gene expression have also been shown to contribute to a wide range of neuropsychiatric disorders, ${ }^{76}$ including mood and anxiety dysregulation. ${ }^{77}$ Based on such findings, and in line with the conceptual approach underlying the recently proposed $\mathrm{NIH}$ Research Domain Criteria ${ }^{78}$ for psychiatric disorders, the correlations between naturally divergent Ahi1 expression and relevant behavioral traits across the BXD RI strains provide a dimensional rather than categorical approach for linking gene expression and behavior.

Although, as we have previously shown, the effect of heterozygous knockout of the Ahi1 gene on protein levels may be limited to the neonatal period, it could still exert longterm consequences, manifested by altered behavior and neural connectivity in adulthood. ${ }^{31}$ This observation is in line with previous data, revealing that in Ahi1-null mice morphological brain anomalies persist into adulthood despite near-normalization of Ahi1-related signal transduction pathways upon maturation of the brain. $^{20}$ The current neuroinformatic analysis consolidates our

Figure 6. Hierarchical organization of whole-brain functional networks. (a-f) Examples of functional networks derived from individual mice held at minimum density $(d=0.34)$. (a, b) Schematic visualizations of dis-hierarchical $(\mathbf{a})$ and hierarchical (b) networks. Node position along the $y$ axis corresponds to nodal degree, while node size corresponds to nodal clustering coefficient. Edges are binarized. Note that, while in the dis-hierarchical network high-degree nodes are also characterized by high clustering coefficients, a reciprocal relationship is evident in the hierarchical network. (c, d) Anatomical visualizations of these same networks, viewed from the horizontal and sagittal planes (top and bottom images, respectively). These images were produced with weighted edges, which depict the strength of the functional connections. Edge colors correspond to the Fisher-transformed Z-score of the correlation coefficient between each pair of connected nodes (see color bar to the right). Only the top $34 \%$ of connections, in terms of their absolute Z-score, are depicted. Nodes are color-coded as in the previous figure. (e, $\mathbf{f}$ ) Heat maps of the adjacency matrices derived from these same networks, based on Z-scores (see color bar). Regions of interest are presented according to their anatomical position along the rostrocaudal axis. $(\mathbf{g}, \mathbf{h})$ The network's hierarchy coefficient $\beta$ is quantified by estimating the inverse of the regression line's slope. $n=13\left(A h i 1^{+/+}\right), n=12\left(A h i 1^{+/-}\right)$. 
previous results by highlighting that, although neocortical Ahi1 expression across strains could significantly predict the efficacy of fear learning in adulthood, the major contribution to this prediction came from the early postnatal expression profile, while the addition of the juvenile and adult profiles did not increase prediction strength. This finding is consistent with current neurodevelopmental theories, suggesting that aberrant expression during the early postnatal period is sufficient to set in motion a cascade of events, which leads to long-lasting changes in brain connectivity and altered anxiety-like behavior throughout life. ${ }^{79,80}$

The QTL analysis utilizing the BXD RI strains, which revealed that a single QTL at the Chr1: 69.048453 locus modulated both early neocortical expression of Ahi1 and fear conditioning in adulthood, bolsters the significant expression-phenotype correlations noted above. ${ }^{75}$ Furthermore, through the identification of additional transcripts whose early neocortical expression is also tightly regulated by the same locus, further insights may be proposed. It has been previously shown that trans-eQTLs associated with expression of diverse genes can often be localized to common loci. ${ }^{81}$ Genes at these loci appear to control the expression of large numbers of downstream genes, suggesting that they act as 'master modulatory loci'. In fact, a key master locus that modulates hundreds of brain transcripts has been identified within the Mtap2 locus, just $1.1 \mathrm{Mb}$ upstream of Ahi1's major trans-eQTL. ${ }^{82}$ Mtap2 contains at least seven missense polymorphisms between C57BL/ $6 \mathrm{~J}$ and $\mathrm{DBA} / 2 \mathrm{~J}$ strains. As Mtap2 is a modestly cis-regulated transcript, these polymorphisms potentially have a causal role in regulating the transcripts mapping to the Chr1: 69.048453 locus during neurodevelopment. Another possibility is that the Chr1: 69.048453 polymorphism, located to ErbB4's first intron, alters the latter's splicing, thereby modulating the expression of relevant downstream genes. Such an idea seems exciting, given the mounting evidence linking dysregulated ErbB4 splicing with abnormal cortical development. ${ }^{83}$

Notably, irrespective of the exact genetic mechanism responsible for the Chr1: 69.048453 trans-regulation of key transcripts, the Gene Ontology categories over-represented among these transcripts include, besides neuron development and differentiation, also the organization of cell projections. Given Ahi1's postulated role in organizing neural projections such as the primary cilium, ${ }^{15,18,19,21,84}$ it is plausible that a trans-acting element at, or adjacent to, the Chr1: 69.048453 locus modulates the expression of a set of genes involved in ciliogenesis. Finally, as this set of genes share suggestive and significant QTLs, other than at Chr1: 69.048453, both among themselves as well as with several fear conditioning traits, preliminary data supporting an association between early neocortical Ahi1 expression, ciliogenesis and fear learning during adulthood are provided.

The placement of brain connectivity as a bridge linking genetics and behavior has been increasingly encouraged. ${ }^{6,78}$ Regarding Ahi1, on the one hand, Ahi1-dependent modulation of Wnt signaling ${ }^{19}$ is presumed to regulate neuronal migration, axonal development and synaptogenesis, ultimately shaping wiring of the brain. ${ }^{85}$ On the other hand, differential Ahi1 expression has been implicated in schizophrenia ${ }^{29}$ and autism ${ }^{30}$ in humans and in distinct cognitive-emotional deficits in mice, ${ }^{31,59,86}$ with convergent findings suggesting that these disorders are associated with specific disturbances of network connectivity. ${ }^{6}$ To this end, while using a seed-voxel analysis of resting-state fMRI data we previously demonstrated that $\mathrm{Ahi}^{+/-}$mice display functional corticolimbic disconnectivity, ${ }^{31}$ a more comprehensive approach to study brain wiring would be to analyze the data in a form of network models. ${ }^{6}$ Thus brain connectivity can be abstracted to a graph of nodes, representing brain regions, linked by edges, representing functional interaction between nodes. Such a representation brings connectomic data into the realm of graph theory, affording a rich repertoire of mathematical tools and concepts that can be used to characterize diverse properties of brain networks. $^{8}$ Specifically, quantifying network topology enables one to generate predictive models regarding the functional consequences attributed to the gene or disorder in question. ${ }^{9}$ Although utilization of graph theory to the study of resting-state functional networks in inbred mice has recently been reported using fMRI, ${ }^{87}$ to the best of our knowledge our current analysis provides the first reported implementation of this approach in the context of genetically modified mouse models.

Originally described in social networks, the 'small-world' property, a prominent measure of global network topology, combines high levels of local clustering with short path lengths that globally link all nodes of the network. Recent brain imaging studies have demonstrated that the human brain's structural and functional networks have small-world properties, ${ }^{4}$ supporting the rapid real-time integration of information across segregated sensory brain regions. These features confer resilience against pathological attacks and maximize efficiency at a minimal cost for effective information processing between different brain regions. ${ }^{88}$ Although reports of resting-state functional networks in rodents are sparse, recent evidence from rats suggests that obtaining small-world coefficients may guarantee that the networks are indeed biologically plausible. ${ }^{89}$ In line with other recent reports, ${ }^{90-92}$ the current fMRI-based graph analysis provides evidence regarding the feasibility of generating resting-state functional networks with small-world properties within the mouse brain. Notably, both $\mathrm{Ahi}^{+/+}$and $\mathrm{Ahi1^{+/- }}$ mice demonstrated small-world properties, most prominent at lower network densities. Nonetheless, small-worldness of brain networks has been a consistent observation, irrespective of illness status, ${ }^{93}$ suggesting that key aspects of brain organization are highly conserved even in the presence of neurodevelopmental disorders with profound effects on complex brain functions. ${ }^{94}$

Our observation that in $\mathrm{Ahi1}^{+/+}$mice whole-brain resting-state functional networks tend to be hierarchical corresponds well with previous studies in humans. ${ }^{95}$ The hubs of hierarchical networks are highly connected but have low clustering; they are mainly connected to nodes that are not otherwise connected to each other. This architecture supports highly efficient information processing and executive-report relationships between nodes while conserving wiring costs. ${ }^{47}$ Moreover, the weighted connectivity graphs and adjacency matrices imply that reciprocal relationships between cortical and non-cortical regions, manifested as negative (anti) correlations, may be more prominent in the hierarchical configuration. In line with recently published data, ${ }^{96,97}$ these findings suggest that hierarchical topology may be associated with a 'division of labor' between several networks. Thus the differential network organization of $\mathrm{Ahi1}^{+/-}$mice, which varied predominantly between non- and dis-hierarchical topologies and lacked significant negative correlations, could underlie their underperformance in tasks relying on efficient information processing. Remarkably, similar findings suggesting lower hierarchy of structural cortical networks have recently been attributed to patients with schizophrenia, ${ }^{94}$ a putatively neurodevelopmental disorder marked by impairments in cognition and information processing, and also associated with AHI1 polymorphisms. ${ }^{24,25}$

The hubs of an assortative network are highly connected to each other. Compared with non-assortative topology, this organization offers robustness against random or targeted removal of hubs. ${ }^{46}$ Our results, showing that on average individual assortativity coefficients in $\mathrm{Ahil}^{+/-}$mice were significantly higher than in $\mathrm{Ahi1}^{+/+}$mice, parallel findings in schizophrenia patients contrasted with healthy volunteers. ${ }^{94}$ These results could imply that functional brain networks in $A h i 1^{+/-}$mice are more resilient when facing pathological attacks. Although in graph theory the term 'attacks' refers to the removal of vertices, acute environmental stress is often seen as an ethologically valid form of a 'behavioral attack'. ${ }^{98}$ Should this conceptual gap between 'attack' nuances in the graph theory vs behavioral fields prove negotiable, 
it could be argued that the $A h i 1^{+/-}$mouse displays a multi-faceted resilient phenotype, manifested in terms of brain network topology as well as behavior. Notably, individual differences in personality traits have recently been associated with topological metrics of whole-brain functional networks. Specifically, the cortical networks of extraverts suggested a configuration that favors a higher arousal threshold and higher levels of arousal tolerance. ${ }^{99}$ Finally, the weighted graphs imply that in the assortative configuration which characterizes the $A$ hi $1^{+/-}$mouse, mesolimbic connections could possibly emerge at the expense of long-distance functional connections. Notably, such a trade-off has been implicated in the pathogenesis of brain disorders that are associated with metabolic distress. ${ }^{88}$

Although global network measures capture important characteristics related to overall efficiency and robustness, it was recently argued that they should be supplemented by the more fine-grained local measures of network topology. ${ }^{100}$ To this end, the data suggesting that functional connectivity of the SC, as assessed by the node-degree parameter, is significantly altered in mice underexpressing the Ahi1 gene, complement the differences observed in global network topology. These midbrain structures have a major role in processing information necessary for spatial locomotion and sensory-guided navigation, ${ }^{101}$ and are also involved in the modulation of avoidance and escape behavior, ${ }^{102}$ all of which are highly relevant components during the swim-to-visible platform task. In this regard, the striking differences between our functional model and wild-type controls in terms of escape latency and thigmotaxis (avoidance of open places), when presented with an immediate sense of danger, could theoretically be explained by a differential layout of visual maps that is caused by subtle differences in Ahi1 expression. Although this speculation obviously needs further validation using direct in vivo recordings, it seems highly attractive, given the recent compelling data implicating a SC-mediated orientation bias in computational processes related to avoidance and escape maneuvers. Specifically, following the hypothesis generated by Ahmadlou and Heimel, ${ }^{60}$ it could be argued that the superior performance displayed by the $\mathrm{Ahi}^{+/-}$mouse in locating the escape platform could be the result of altered gaze towards, and/ or detection of, critical visual stimuli located in the arena center, while the mouse engages in thigmotactic radial motion. To this end, and although direct evidence linking Ahi1 expression with layering of the SC has not been examined, preliminary support for such a role could be drawn from data based on a different Ahi1-related functional model, which suggests that in utero induction of Ahi1 overexpression leads to abnormal cortical layering (unpublished data).

The current work uses different methodological approaches to unravel the neurodevelopmental mechanisms that are under study. Thus behavioral experiments in rodents are complemented by functional imaging and graph-theory-based analysis. Moreover, we took advantage of comprehensive online data sets to seek support for some of our key findings. ${ }^{103}$ Thereby, we were able to corroborate and extend our original functional data. Moreover, the approach employed in the construction of large expression data sets, such as those provided by GeneNetwork, ${ }^{39}$ treats gene expression as a continuous variable across RI strains, rather than as a categorical one (knockout model). Hence, we believe that using these complementary, yet conceptually distinct, approaches enhanced our ability to propose mechanistic insights.

A limitation of the current study relates to the non-trivial relationship between structural and functional brain connectivity. ${ }^{4}$ Structural networks represent anatomical wiring diagrams. In contrast, functional networks represent patterns of activity correlations that do not necessarily coincide with direct neuronal communication. Although assuming a role for Ahi1 in emerging structural connections seems relatively straightforward, given its postulated role in neuronal migration and primary cilia development, the current graph-based analysis is based on measures of functional connectivity. Nonetheless, convergent findings indicate that the brain's structural and functional networks are intimately related and share common topological features. ${ }^{4,96}$ Moreover, Ahi1 could also directly modulate the strengths of functional connections through its putative roles in ciliogenesis $^{84}$ and Wnt-mediated synaptogenesis. ${ }^{104}$ Supporting this hypothesis, recent evidence suggests that loss of Ahi1 impairs neurotransmitter release. ${ }^{59}$ Future studies conducting topological comparisons between anatomical and functional connections will help delineate the mechanisms through which Ahi1 affects wiring of the brain. Another possible shortcoming related to the generation of functional networks is the relatively small number of nodes used. However, while networks that are comprised of hundreds, or even thousands, of nodes may be ideal for quantifying global metrics and for the detection of subtle between-group differences, basic configurations can also be readily visualized in networks comprised of only several dozens of nodes. ${ }^{47,94}$ Nevertheless, replication of our findings using larger networks is warranted and should be made feasible in the near future given the tremendous advances in imaging technology. Finally, the use of isoflurane anesthesia during acquisition of functional data has recently been debated. However, a recent report suggested that, even though local functional properties could become reorganized under isoflurane anesthesia in rats, global topological features were preserved. ${ }^{105}$ Moreover, another recent report indicating that the use of isoflurane does not mask network information ${ }^{43}$ bolsters the validity of our methodology.

A major limitation of the current study is the lack of direct functional data assessing the long-term effects of reduced Ahi1 expression in specific brain regions and during specific developmental stages. Investigating the long-term effects exerted by temporal expression profiles of specific genes has been the focus of several studies employing heterozygous knockout models for neurodevelopmental genes. For instance, mice with heterozygous knockout of GAP-43, a gene involved in some forms of neural plasticity, ${ }^{106}$ display a significant decrease in the corresponding protein levels in newborns, with a gradual convergence to nearnormal protein levels in later life. ${ }^{107}$ In line with this observation, we had previously shown that in our current functional model, Ahi1 protein levels in the adult mouse brain did not differ between wild types and Ahi1 heterozygous knockouts. ${ }^{31}$ However, in neonates, when Ahi1 expression is presumably at its peak, ${ }^{15,74}$ protein levels were significantly lower in the $\mathrm{Ahi1}^{+/}$mice. Thus we argue that our model does recapitulate, albeit to a limited extent, the 'essence' of temporal conditionality, as the striking differences in both network topology and behavior during adulthood could be demonstrated (long) after the initial differences in Ahi1 expression had already subsided. Moreover, while manipulating Ahi1 expression across several regions would be a most informative approach to delineating the spatial roles of Ahi1, we argue that the data suggesting that functional connectivity of the SC is significantly altered in mice underexpressing the Ahi1 gene provide additional evidence linking alterations in Ahi1 expression with spatial phenotypes and possibly also with visually based information processing and behavior. Nonetheless, future generation of spatiotemporal conditional knockout models is highly warranted, as it will likely stimulate new insights into Ahi1-related cognitive and emotional developmental trajectories.

Finally, although we have attempted to depict a cascade linking gene expression, brain wiring and behavior through multiple lines of evidence, a phenotype at the neuronal level could provide another important link. However, while full Ahi1 knockout results in striking histological phenotypes, ${ }^{20}$ in our mouse model, which is based on moderate reduction of Ahi1 expression, we expect neuronal phenotypes to be subtle. To this end, and given Ahi1's postulated role in the assembly of subcellular components such as 
the primary cilium, ${ }^{19}$ together with the data from the eQTL-based enrichment analysis, electron microscopic studies in neuronal cultures could shed valuable light on further links in this developmental cascade.

A central idea emanating from the current work is that physiological variation in the expression of genes that are coregulated and function together during neurodevelopment may eventually be translated into distinct intermediate phenotypes along a continuum of metrics depicting network topology. Within this framework, it seems that the brain's connectome may not be optimized to maximize advantageous topological properties, ${ }^{88}$ such as hierarchy or assortativity. Instead, we propose that brain network organization could be the result of an adaptive trade-off between efficiency of the brain network and its resilience. Ultimately, and possibly a consequence of the former, a compound behavioral phenotype along a cognitive-emotional continuum may crystallize, expressing varying levels of cognitive performance and stress resilience. Extrapolating from this hypothesis, and in line with recent theoretical models, ${ }^{88}$ it could be proposed that impairments in cognitive function and/or emotional regulation that characterize neuropsychiatric disorders may reflect genetically mediated effects on those global properties of brain networks that are crucial for integrative processing and adaptive behavior.

\section{CONFLICT OF INTEREST}

The authors declare no conflict of interest.

\section{ACKNOWLEDGMENTS}

Supported in part by a grant from the Israel Science Foundation (ISF) (ISF-NSFC Joint Scientific Research Program) (to BL). We thank Dr R Williams, Dr A Heimel and Dr R Overall for their teaching during the INCF Short Course on Neuroinformatics, Neurogenomics and Brain Disease (to AL). We thank Dr Glenn Rosen for generating the developmental data sets that have been so useful for the current analysis. We thank $\operatorname{Dr}$ A Rigbi for his assistance in the statistical analysis of the data.

\section{REFERENCES}

1 Van Den Bergh BRH. Developmental programming of early brain and behaviour development and mental health: a conceptual framework. Dev Med Child Neurol 2011; 53: 19-23.

2 Koten JW, Wood G, Hagoort P, Goebel R, Propping P, Willmes K et al. Genetic contribution to variation in cognitive function: an $\mathrm{fMRl}$ study in twins. Science 2009; 323: 1737-1740.

3 Schmitt JE, Lenroot RK, Wallace GL, Ordaz S, Taylor KN, Kabani N et al. Identification of genetically mediated cortical networks: a multivariate study of pediatric twins and siblings. Cereb Cortex 2008; 18: 1737-1747.

4 Bullmore E, Sporns O. Complex brain networks: graph theoretical analysis of structural and functional systems. Nat Rev Neurosci 2009; 10: 186-198.

5 Rubinov M, Sporns O. Complex network measures of brain connectivity: uses and interpretations. Neurolmage 2010; 52: 1059-1069.

6 Sporns 0 . Contributions and challenges for network models in cognitive neuroscience. Nat Neurosci 2014; 17: 652-660.

7 Marcus D, Harwell J, Olsen T, Hodge M, Glasser M, Prior F et al. Informatics and data mining tools and strategies for the Human Connectome Project. Front Neuroinform 2011; 5: 4.

8 Fornito A, Zalesky A, Breakspear M. Graph analysis of the human connectome: promise, progress, and pitfalls. Neurolmage 2013; 80: 426-444.

9 Fornito A, Zalesky A, Breakspear M. The connectomics of brain disorders. Nat Rev Neurosci 2015; 16: 159-172.

10 Sporns O. Structure and function of complex brain networks. Dialogues Clin Neurosci 2013; 15: 247-262.

11 Arguello PA, Gogos JA. Genetic and cognitive windows into circuit mechanisms of psychiatric disease. Trends Neurosci 2012; 35: 3-13.

12 Pratt J, Winchester C, Dawson N, Morris B. Advancing schizophrenia drug discovery: optimizing rodent models to bridge the translational gap. Nat Rev Drug Discov 2012; 11: 560-579.

13 Schughart K, Libert C, Kas MJ. Human disease: strength to strength for mouse models. Nature 2012; 492: 41-41.
14 Sporns O, Bullmore Edward T. From connections to function: the Mouse Brain Connectome Atlas. Cell 2014; 157: 773-775.

15 Doering J, Kane K, Hsiao Y, Yao C, Shi B, Slowik A et al. Species differences in the expression of Ahi1, a protein implicated in the neurodevelopmental disorder Joubert syndrome, with preferential accumulation to stigmoid bodies. J Comp Neurol 2008; 511: 238-256.

16 Yu F, Keinan A, Chen H, Ferland R, Hill R, Mignault A et al. Detecting natural selection by empirical comparison to random regions of the genome. Hum $\mathrm{Mol}$ Genet 2009; 18: 4853-4867.

17 Louie C, Caridi G, Lopes V, Brancati F, Kispert A, Lancaster M et al. AHI1 is required for photoreceptor outer segment development and is a modifier for retinal degeneration in nephronophthisis. Nat Genet 2010; 42: 175-180.

18 Westfall JE, Hoyt C, Liu Q, Hsiao YC, Pierce EA, Page-McCaw PS et al. Retinal degeneration and failure of photoreceptor outer segment formation in mice with targeted deletion of the Joubert syndrome gene, Ahi1. J Neurosci 2010; 30: 8759-8768.

19 Lancaster MA, Schroth J, Gleeson JG. Subcellular spatial regulation of canonical Wnt signalling at the primary cilium. Nat Cell Biol 2011; 13: 700-707.

20 Lancaster MA, Gopal DJ, Kim J, Saleem SN, Silhavy JL, Louie CM et al. Defective Wnt-dependent cerebellar midline fusion in a mouse model of Joubert syndrome. Nat Med 2011; 17: 726-731.

21 Tuz K, Hsiao YC, Juarez O, Shi B, Harmon EY, Phelps IG et al. The Joubert syndrome-associated missense mutation (V443D) in the Abelson-helper integration site 1 (AHI) protein alters its localization and protein-protein interactions. J Biol Chem 2013; 288: 13676-13694.

22 Weng L, Lin YF, Li AL, Wang CE, Yan S, Sun M et al. Loss of Ahi1 affects early development by impairing BM88/Cend1-mediated neuronal differentiation. J Neurosci 2013; 33: 8172-8184.

23 Lerer B, Segman R, Hamdan A, Kanyas K, Karni O, Kohn Y et al. Genome scan of Arab Israeli families maps a schizophrenia susceptibility gene to chromosome $6 q 23$ and supports a locus at chromosome 10q24. Mol Psychiatry 2003; 8: 488-498.

24 Amann-Zalcenstein D, Avidan N, Kanyas K, Ebstein R, Kohn Y, Hamdan A et al. AHI1, a pivotal neurodevelopmental gene, and C6orf217 are associated with susceptibility to schizophrenia. Eur J Hum Genet 2006; 14: 1111-1119.

25 Ingason A, Giegling I, Cichon S, Hansen T, Rasmussen H, Nielsen J et al. A large replication study and meta-analysis in European samples provides further support for association of AHI1 markers with schizophrenia. Hum Mol Genet 2010; 19: 1379-1386.

26 Rivero O, Reif A, Sanjuán J, Moltó M, Kittel-Schneider S, Nájera C et al. Impact of the AHI1 gene on the vulnerability to schizophrenia: a case-control association study. PLoS One 2010; 5: e12254.

27 Alvarez Retuerto A, Cantor R, Gleeson J, Ustaszewska A, Schackwitz W, Pennacchio $L$ et al. Association of common variants in the Joubert syndrome gene (AHI1) with autism. Hum Mol Genet 2008; 17: 3887-3896.

28 Porcelli S, Pae C-U, Han C, Lee S-J, Patkar AA, Masand PS et al. Abelson helper integration site-1 gene variants on major depressive disorder and bipolar disorder. Psychiatry Investig 2014; 11: 481-486.

29 Slonimsky A, Levy I, Kohn Y, Rigbi A, Ben-Asher E, Lancet D et al. Lymphoblast and brain expression of AHI1 and the novel primate-specific gene, C6orf217, in schizophrenia and bipolar disorder. Schizophr Res 2010; 120: 159-166.

30 Voineagu I, Wang X, Johnston P, Lowe JK, Tian Y, Horvath S et al. Transcriptomic analysis of autistic brain reveals convergent molecular pathology. Nature 2011; 474: $380-384$.

31 Lotan A, Lifschytz T, Slonimsky A, Broner EC, Greenbaum L, Abedat S et al. Neural mechanisms underlying stress resilience in Ahi1 knockout mice: relevance to neuropsychiatric disorders. Mol Psychiatry 2014; 19: 243-252.

32 Lotan A, Lifschytz T, Lory O, Goelman G, Lerer B. Amygdalar disconnectivity could underlie stress resilience in the Ahi1 knockout mouse: conclusions from a resting-state functional MRI study. Mol Psychiatry 2014; 19: 144.

33 Chesler EJ, Lu L, Wang J, Williams RW, Manly KF. WebQTL: rapid exploratory analysis of gene expression and genetic networks for brain and behavior. Nat Neurosci 2004; 7: 485-486.

34 Skarnes WC, von Melchner H, Wurst W, Hicks G, Nord AS, Cox T et al. A public gene trap resource for mouse functional genomics. Nat Genet 2004; 36: 543-544.

35 Cheng YZ, Eley L, Hynes AM, Overman LM, Simms RJ, Barker A et al. Investigating embryonic expression patterns and evolution of AHI1 and CEP290 genes, implicated in Joubert syndrome. PLoS One 2012; 7: e44975.

36 Beiko J, Lander R, Hampson E, Boon F, Cain DP. Contribution of sex differences in the acute stress response to sex differences in water maze performance in the rat. Behav Brain Res 2004; 151: 239-253.

37 Bevins RA, Besheer J. Object recognition in rats and mice: a one-trial nonmatching-to-sample learning task to study 'recognition memory'. Nat Protoc 2006; 1: 1306-1311. 
38 Licht T, Goshen I, Avital A, Kreisel T, Zubedat S, Eavri R et al. Reversible modulations of neuronal plasticity by VEGF. Proc Natl Acad Sci 2011; 108: 5081-5086.

39 Wang J, Williams R, Manly K. WebQTL. Neuroinformatics 2003; 1: 299-308.

40 Taylor BA, Wnek C, Kotlus BS, Roemer N, MacTaggart T, Phillips SJ. Genotyping new BXD recombinant inbred mouse strains and comparison of BXD and consensus maps. Mamm Genome 1999; 10: 335-348.

41 Geisert EE, Lu L, Freeman-Anderson NE, Templeton JP, Nassr M, Wang X et al. Gene expression in the mouse eye: an online resource for genetics using 103 strains of mice. Mol Vis 2009; 15: 1730-1763.

42 Pandey AK, Williams RW. Genetics of gene expression in CNS. Int Rev Neurobiol 2014; 116: 195-231.

43 Goelman G, llinca R, Zohar I, Weinstock M. Functional connectivity in prenatally stressed rats with and without maternal treatment with ladostigil, a brainselective monoamine oxidase inhibitor. Eur J Neurosci 2014; 40: 2734-2743.

44 Paxinos G, Franklin KBJ. The Mouse Brain in Stereotaxic Coordinates. Compact2nd edn.Elsevier Academic Press: Amsterdam, the Netherlands; Boston, MA, USA, 2004

45 Latora V, Marchiori M. Efficient behavior of small-world networks. Phys Rev Lett 2001; 87: 19.

46 Newman MEJ. Assortative mixing in networks. Phys Rev Lett 2002; 89: 20.

47 Ravasz E, Barabási A-L. Hierarchical organization in complex networks. Phys Rev $E$ 2003; 67: 026112.

48 Hosseini SM, Hoeft F, Kesler SR. GAT: a graph-theoretical analysis toolbox for analyzing between-group differences in large-scale structural and functional brain networks. PLoS One 2012; 7: e40709.

49 Tybout A, Sternthal B, Keppel G, Verducci J, Meyers-Levy J, Barnes J et al. Analysis of variance. J Consum Psychol 2001; 10: 5-35.

50 Besheer J, Bevins RA. Object recognition in rats and mice: a one-trial nonmatching-to-sample learning task to study 'recognition memory'. Nat Protoc 2006; 1: 1306-1311.

51 Johansen Joshua P, Cain Christopher K, Ostroff Linnaea E, LeDoux Joseph E. Molecular mechanisms of fear learning and memory. Cell 2011; 147: 509-524.

52 Curzon P, Rustay NR, Browman KECued and contextual fear conditioning for rodentsInBuccafusco JJ(ed). Methods of Behavior Analysis in Neuroscience, 2nd edn 2009.

53 Owen EH, Christensen SC, Paylor R, Wehner JM. Identification of quantitative trait loci involved in contextual and auditory-cued fear conditioning in BXD recombinant inbred strains. Behav Neurosci 1997; 111: 292-300.

54 Philip VM, Duvvuru S, Gomero B, Ansah TA, Blaha CD, Cook MN et al. Highthroughput behavioral phenotyping in the expanded panel of BXD recombinant inbred strains. Genes Brain Behav 2010; 9: 129-159.

55 Brigman JL, Mathur P, Lu L, Williams RW, Holmes A. Genetic relationship between anxiety-related and fear-related behaviors in BXD recombinant inbred mice. Behav Pharmacol 2009; 20: 204-209.

56 Yang RJ, Mozhui K, Karlsson R-M, Cameron HA, Williams RW, Holmes A. Variation in mouse basolateral amygdala volume is associated with differences in stress reactivity and fear learning. Neuropsychopharmacology 2008; 33: 2595-2604.

57 Sheng G, Xu X, Lin Y, Wang C, Rong J, Cheng D et al. Huntingtin-associated protein 1 interacts with Ahi1 to regulate cerebellar and brainstem development in mice. J Clin Invest 2008; 118: 2785-2795.

58 Xu X, Yang H, Lin YF, Li X, Cape A, Ressler KJ et al. Neuronal Abelson helper integration site-1 (Ahi1) deficiency in mice alters TrkB signaling with a depressive phenotype. Proc Natl Acad Sci USA 2010; 107: 19126-19131.

59 Ren L, Qian X, Zhai L, Sun M, Miao Z, Li J et al. Loss of Ahi1 impairs neurotransmitter release and causes depressive behaviors in mice. PLoS One 2014; 9: e93640.

60 Ahmadlou M, Heimel JA. Preference for concentric orientations in the mouse superior colliculus. Nat Commun 2015; 6: 6773.

61 Crawley JN. Exploratory behavior models of anxiety in mice. Neurosci Biobehav Rev 1985; 9: 37-44.

62 Treit D, Fundytus M. Thigmotaxis as a test for anxiolytic activity in rats. Pharmacol Biochem Behav 1988; 31: 959-962.

63 Kant GJ, Lenox RH, Bunnell BN, Mougey EH, Pennington LL, Meyerhoff JL. Comparison of stress response in male and female rats: pituitary cyclic AMP and plasma prolactin, growth hormone and corticosterone. Psychoneuroendocrinology 1983; 8: 421-428.

64 Ennaceur A, Delacour J. A new one-trial test for neurobiological studies of memory in rats. 1: Behavioral data. Behav Brain Res 1988; 31: 47-59.

65 Akkerman S, Blokland A, Reneerkens O, van Goethem NP, Bollen E, Gijselaers HJM et al. Object recognition testing: methodological considerations on exploration and discrimination measures. Behav Brain Res 2012; 232: 335-347.

66 Conti L, Maciver C, Ferkany J, Abreu M. Footshock-induced freezing behavior in rats as a model for assessing anxiolytics. Psychopharmacology (Berl) 1990; 102: 492-497.
67 Sato M, Ito M, Nagase M, Sugimura Y, Takahashi Y, Watabe A et al. The lateral parabrachial nucleus is actively involved in the acquisition of fear memory in mice. Mol Brain 2015; 8: 22.

68 Botterill JJ, Fournier NM, Guskjolen AJ, Lussier AL, Marks WN, Kalynchuk LE. Amygdala kindling disrupts trace and delay fear conditioning with parallel changes in Fos protein expression throughout the limbic brain. Neuroscience 2014; 265: 158-171.

69 Dickerson BC, Eichenbaum H. The episodic memory system: neurocircuitry and disorders. Neuropsychopharmacology 2009; 35: 86-104.

70 Levy RJ, Kvajo M, Li Y, Tsvetkov E, Dong W, Yoshikawa Y et al. Deletion of Rapgef6, a candidate schizophrenia susceptibility gene, disrupts amygdala function in mice. Transl Psychiatry 2015; 5: e577.

71 Lissek S, Powers AS, McClure EB, Phelps EA, Woldehawariat G, Grillon C et al. Classical fear conditioning in the anxiety disorders: a meta-analysis. Behav Res Ther 2005; 43: 1391-1424.

72 Peri T, Ben-Shakhar G, Orr SP, Shalev AY. Psychophysiologic assessment of aversive conditioning in posttraumatic stress disorder. Biol Psychiatry 2000; 47: 512-519.

73 Dixon-Salazar T, Silhavy J, Marsh S, Louie C, Scott L, Gururaj A et al. Mutations in the AHI1 gene, encoding jouberin, cause Joubert syndrome with cortical polymicrogyria. Am J Hum Genet 2004; 75: 979-987.

74 Ferland R, Eyaid W, Collura R, Tully L, Hill R, Al-Nouri D et al. Abnormal cerebellar development and axonal decussation due to mutations in AHI1 in Joubert syndrome. Nat Genet 2004; 36: 1008-1013.

75 Wang X, Pandey AK, Mulligan MK, Williams EG, Mozhui K, Li Z et al. Joint mousehuman phenome-wide association to test gene function and disease risk. Nat Commun 2016; 7: 10464.

76 Lotan A, Fenckova M, Bralten J, Alttoa A, Dixson L, Williams R et al. Neuroinformatic analyses of common and distinct genetic components associated with major neuropsychiatric disorders. Front Neurosci 2014; 8: 331.

77 Knight JC. Regulatory polymorphisms underlying complex disease traits. J Mol Med 2005; 83: 97-109.

78 Morris SE, Cuthbert BN. Research domain criteria: cognitive systems, neural circuits, and dimensions of behavior. Dialogues Clin Neurosci 2012; 14: 29-37.

79 Gross C, Zhuang X, Stark K, Ramboz S, Oosting R, Kirby L et al. Serotonin1A receptor acts during development to establish normal anxiety-like behaviour in the adult. Nature 2002; 416: 396-400.

80 Lenze EJ, Wetherell JL. A lifespan view of anxiety disorders. Dialogues Clin Neurosci 2011; 13: 381-399.

81 Overall RW, Kempermann G, Peirce J, Lu L, Goldowitz D, Gage FH et al. Genetics of the hippocampal transcriptome in mouse: a systematic survey and online neurogenomics resource. Front Neurosci 2009; 3: 55.

82 Chesler EJ, Lu L, Shou S, Qu Y, Gu J, Wang J et al. Complex trait analysis of gene expression uncovers polygenic and pleiotropic networks that modulate nervous system function. Nat Genet 2005; 37: 233-242.

83 Chung DW, Volk DW, Arion D, Zhang Y, Sampson AR, Lewis DA. Dysregulated ErbB4 splicing in schizophrenia: selective effects on parvalbumin expression. Am J Psychiatry 173: 60-68.

84 Hsiao Y, Tong Z, Westfall J, Ault J, Page-McCaw P, Ferland R. Ahi1 whose human ortholog is mutated in Joubert syndrome, is required for Rab8a localization, ciliogenesis and vesicle trafficking. Hum Mol Genet 2009; 18: 3926-3941.

85 Salinas PC, Zou Y. Wnt signaling in neural circuit assembly. Annu Rev Neurosci 2008; 31: 339-358.

86 Xu X, Yang H, Lin YF, Li X, Cape A, Ressler KJ et al. Neuronal Abelson helper integration site-1 (Ahi1) deficiency in mice alters TrkB signaling with a depressive phenotype. Proc Natl Acad Sci USA 2010; 107: 19126-19131.

87 Mechling $A E$, Hübner NS, Lee $\mathrm{H}-\mathrm{L}$, Hennig J, von Elverfeldt D, Harsan L-A. Finegrained mapping of mouse brain functional connectivity with resting-state fMRI. Neurolmage 2014; 96: 203-215.

88 Bullmore E, Sporns O. The economy of brain network organization. Nat Rev Neurosci 2012; 13: 336-349.

89 Baliki MN, Chang PC, Baria AT, Centeno MV, Apkarian AV. Resting-sate functional reorganization of the rat limbic system following neuropathic injury. Sci Rep 2014; 4: 6186

90 Dawson N, McDonald M, Higham DJ, Morris BJ, Pratt JA. Subanesthetic ketamine treatment promotes abnormal interactions between neural subsystems and alters the properties of functional brain networks. Neuropsychopharmacology 2014; 39: 1786-1798.

91 Dawson N, Kurihara M, Thomson DM, Winchester CL, McVie A, Hedde JR et al. Altered functional brain network connectivity and glutamate system function in transgenic mice expressing truncated Disrupted-in-Schizophrenia 1. Transl Psychiatry 2015; 5: e569.

92 Dawson N, Xiao X, McDonald M, Higham DJ, Morris BJ, Pratt JA. Sustained NMDA receptor hypofunction induces compromised neural systems integration and schizophrenia-like alterations in functional brain networks. Cereb Cortex 2014; 24: 452-464. 
93 Zhao X, Liu Y, Wang X, Liu B, Xi Q, Guo Q et al. Disrupted small-world brain networks in moderate Alzheimer's disease: a resting-state fMRI study. PLoS One 2012; 7: e33540.

94 Bassett DS, Bullmore E, Verchinski BA, Mattay VS, Weinberger DR, MeyerLindenberg A. Hierarchical organization of human cortical networks in health and schizophrenia. J Neurosci 2008; 28: 9239-9248.

95 Liang X, Wang J, Yan C, Shu N, Xu K, Gong G et al. Effects of different correlation metrics and preprocessing factors on small-world brain functional networks: a resting-state functional MRI study. PLoS One 2012; 7: e32766.

96 Hosseini SMH, Kesler SR. Comparing connectivity pattern and small-world organization between structural correlation and resting-state networks in healthy adults. Neurolmage 2013; 78: 402-414.

97 Goelman G, Gordon N, Bonne O. Maximizing negative correlations in resting-state functional connectivity MRI by time-lag. PLoS One 2014; 9: e111554.

98 Buccafusco JJ, Buccafusco JJ. Methods of Behavioral Analysis in Neuroscience2nd edn.CRC Press: Boca Raton, FL, USA, 2009 xxi, 351 pp.

99 Gao Q, Xu Q, Long Z, Duan X, Liao W, Ding J et al. Extraversion and neuroticism relate to topological properties of resting-state brain networks. Front Hum Neurosci 2013; 7: 257.

100 Zalesky A, Fornito A, Bullmore ET. Network-based statistic: identifying differences in brain networks. Neurolmage 2010; 53: 1197-1207.

101 Felsen G, Mainen ZF. Neural substrates of sensory-guided locomotor decisions in the rat superior colliculus. Neuron 2008; 60: 137-148.
102 Dean P, Redgrave P, Westby GWM. Event or emergency? Two response systems in the mammalian superior colliculus. Trends Neurosci 1989; 12: 137-147.

103 Steegen S, Dewitte L, Tuerlinckx F, Vanpaemel W. Measuring the crowd within again: a pre-registered replication study. Front Psychol 2014; 5: 786.

104 Gogolla N, Galimberti I, Deguchi Y, Caroni P. Wnt signaling mediates experiencerelated regulation of synapse numbers and mossy fiber connectivities in the adult hippocampus. Neuron 2009; 62: 510-525.

105 Liang Z, King J, Zhang N. Intrinsic organization of the anesthetized brain. J Neurosci 2012; 32: 10183-10191.

106 Frey D, Laux T, Xu L, Schneider C, Caroni P. Shared and unique roles of Cap23 and Gap43 in actin regulation, neurite outgrowth, and anatomical plasticity. J Cell Biol 2000; 149: 1443-1454.

107 Mcllvain V, McCasland JS. GAP-43 heterozygous mice show delayed barrel patterning, differentiation of radial glia, and downregulation of GAP-43. Anat Rec $A$ Discov Mol Cell Evol Biol 2006; 288A: 143-157.

(c)(2) This work is licensed under a Creative Commons Attributioncc. ${ }_{\text {BY }}$ NC SA NonCommercial-ShareAlike 4.0 International License. The images or other third party material in this article are included in the article's Creative Commons license, unless indicated otherwise in the credit line; if the material is not included under the Creative Commons license, users will need to obtain permission from the license holder to reproduce the material. To view a copy of this license, visit http:// creativecommons.org/licenses/by-nc-sa/4.0/

Supplementary Information accompanies the paper on the Molecular Psychiatry website (http://www.nature.com/mp) 